CLNS 91/1059

April 1991 (revised)

\title{
Structure Constants of the Fractional Supersymmetry Chiral Algebras
}

\author{
Philip C. Argyres ${ }^{\star}$, James M. Grochocinski ${ }^{\dagger}$, And S.-H. Henry Tye \\ Newman Laboratory of Nuclear Studies \\ Cornell University \\ Ithaca, N.Y. 14853-5001
}

\begin{abstract}
The fractional supersymmetry chiral algebras, $\mathcal{A}^{(K)}$, in two-dimensional conformal field theory are extended Virasoro algebras with fractional spin currents $J^{(K)}$. We show that associativity and closure of $\mathcal{A}^{(K)}$ determines its structure constants in the case that the Virasoro algebra is extended by precisely one current. We compute the structure constants of the $\mathcal{A}^{(K)}$ algebras explicitly and we show that correlators of $J^{(K)}$ 's satisfy non-Abelian braiding relations.
\end{abstract}

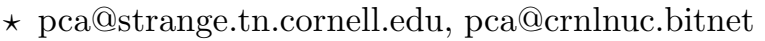

† james@beauty.tn.cornell.edu, james@crnlnuc.bitnet
} 


\section{Introduction}

Extended chiral algebras (algebras which include the Virasoro algebra as a subalgebra) are the main tool for organizing representations and classifying models in two-dimensional conformal field theory (CFT). They also constitute the basis of attempts to form new "string-like" theories by gauging these chiral algebras. For both CFT and string applications, the chiral algebras must be associative operator algebras. This condition places tight constraints on the structure constants and operator content of the extended Virasoro algebra. The structure constants of many chiral algebras have been determined in this way $[1,2,3,4]$. The goal of this paper is to do the same for the fractional supersymmetry chiral algebras. These are non-local algebras which enlarge the Virasoro symmetry algebra to include a fractional spin current. In quantum field theory, it is well known that fractional spin fields satisfy non-trivial braiding relations. In the two-dimensional fractional supersymmetry chiral algebras that we will consider, some of the currents have scaling dimensions other than (half) integers. Since the currents are chiral fields, the fact that they have fractional dimensions implies that they also have fractional spins. The correlators of these currents obey non-Abelian braiding relations. In this paper we show how such braiding relations can be disentangled.

There is a large body of evidence that fractional supersymmetry chiral algebras organize infinite sets of CFTs in the same way that local extended Virasoro algebras do. These algebras were conjectured $[5,6,7]$ in the context of $S U(2)$ coset constructions, and were presented as a new way of organizing $1<c<3$ representations in CFT. Using a BRST cohomology approach [8] based on the fractional supersymmetry algebras, some details of the coset models have been worked out [9], while new coset models have been constructed [10]. These new coset models are $S U(2)_{K} \otimes S U(2)_{L} / S U(2)_{K+L}$ where both $K$ and $L$ are rational; use of the fractional supersymmetry chiral algebras enables one to construct their branching

functions. Another body of evidence comes from the study of perturbed CFT and massive integrable systems, where fractional supersymmetry chiral algebras 
play an important role $[11,12]$. More recently, the fractional supersymmetry chiral algebras have also been proposed $[13,14]$ as the basis for fractional superstrings, generalizing the bosonic string and the superstring. For all these applications it is important to determine the structure constants of the fractional supersymmetry chiral algebras - indeed, without them the chiral algebras and their representation theory are not fully defined.

In general, the fractional supersymmetry chiral algebras are generated by the energy-momentum tensor and a set of fractional spin currents $J_{i}(z)$. For the $K$-th fractional supersymmetry algebra, the $J_{i}$ must have dimension $(K+4) /(K+2)$. In addition, closure of the operator algebra may require that chiral fields with other fractional dimensions enter in the fractional supersymmetry chiral algebra. In this paper, we will assume that there is only one fractional spin current. This is not necessarily the most interesting case, but serves as an example of our approach to the problem of constructing fractional supersymmetry chiral algebras. Our approach is equally applicable to the more general case, although the analysis clearly becomes more involved.

The fractional supersymmetry algebras will be denoted $\mathcal{A}^{(K)}$. The fractional spin of the currents is reflected in the appearance of fractional powers in the $\mathcal{A}^{(K)}$ operator product expansions (OPEs). They are generated by two currents: the energy-momentum tensor $T(z)$ and the fractional supersymmetry current $J^{(K)}(z)$, with the following OPEs

$$
\begin{aligned}
T(z) T(w) & =\frac{c}{2}(z-w)^{-4}\{1+\ldots\}, \\
T(z) J^{(K)}(w) & =\Delta(z-w)^{-2}\left\{J^{(K)}(w)+\ldots\right\}, \\
J^{(K)}(z) J^{(K)}(w) & =(z-w)^{-2 \Delta}\{1+\ldots\}+\lambda_{K}(c)(z-w)^{-\Delta}\left\{J^{(K)}(w)+\ldots\right\},
\end{aligned}
$$

where $\Delta=(K+4) /(K+2)$ is the dimension of $J^{(K)}$, and where the dots represent the Virasoro descendants. The existence of the current $J^{(K)}$ was proposed earlier $[5,6,7]$. However, the existence of the algebra (1.1) can be established only if the 
coupling $\lambda_{K}$ can be consistently determined. The main result of this paper is to show that the fractional supersymmetry algebra is consistent with $\lambda_{K}$ given by

$$
\lambda_{K}^{2}(c)=\frac{2 K^{2}\left(c_{111}\right)^{2}}{3(K+4)(K+2)}\left[\frac{3(K+4)^{2}}{K(K+2)} \frac{1}{c}-1\right]
$$

where, with $\rho=\frac{1}{K+2}$,

$$
\left(c_{111}\right)^{2}=\frac{\sin ^{2}(\pi \rho) \sin ^{2}(4 \pi \rho)}{\sin ^{3}(2 \pi \rho) \sin (3 \pi \rho)} \frac{\Gamma^{3}(\rho) \Gamma^{2}(4 \rho)}{\Gamma(3 \rho) \Gamma^{4}(2 \rho)}
$$

is the chiral structure constant for three spin-1 primaries in the $S U(2)_{K}$ WessZumino-Witten (WZW) theory. The result (1.2) was obtained earlier [13] but without determining $c_{111}$. It is an important point of this paper that $c_{111}$ does not equal the usual $S U(2)_{K}$ structure constants computed in non-chiral theories combining left- and right-moving sectors $[15,16]$. Notice that $\lambda_{K}$ vanishes when $K=2 ;$ this corresponds to the superVirasoro algebra where the supercurrent $J^{(K)}$ has dimension $3 / 2$. Also, for $K=4$, we recover the known structure constant for (a diagonal version of) the spin-4/3 algebra [4].

At this point the reader may worry that if the above chiral algebras have different structure constants than those required in a non-chiral theory, then how can these chiral algebras organize non-chiral (local) CFTs? Indeed, the existence of non-local chiral algebras in two-dimensional CFT is problematical in general because the locality of the full (left plus right) correlation functions rules out the appearance of the fractional spin currents as fields in the full CFT. Nevertheless, it is found that the Ward identities following from such chiral algebras, even though they involve chiral structure constants and unphysical correlation functions with cuts on the world-sheet, do relate physical correlation functions of non-chiral theories, and thus serve as organizing symmetries of local CFTs. The apparent contradiction between the chiral and non-chiral structure constants is avoided because the fractional spin current associated with the chiral structure constants never appears in physical correlators, but only in the derivation of the Ward identities relating 
physical correlators. The structure constants and correlation functions for sets of CFTs have been worked out in this way for the two simplest fractional supersymmetry chiral algebras - the superVirasoro algebra and the spin-4/3 algebra $[17,4,18]$. The evidence from $S U(2)$ coset models and from two-dimensional integrable models, mentioned above, suggests that a similar picture should be true of the other fractional supersymmetry chiral algebras. This paper is intended as a first step in the exploration of fractional spin chiral algebras. In particular, we will not be able to throw much light on precisely how a local (non-chiral) two-dimensional CFT is interpreted as a representation of a non-local chiral algebra. Instead, we will concentrate on the construction and properties of the chiral algebras themselves, without addressing the problem of their representation theory.

The $\lambda_{K}$ found in (1.2) is determined by imposing two consistency conditions, namely associativity and closure. By the closure condition, we mean that only the identity and $J^{(K)}$ and their Virasoro descendants are allowed in the chiral algebra: no new primary operators with different dimensions can appear in (1.1), even with positive fractional powers of $(z-w)$, since such terms introduce new cuts in the $z$ plane. The determination of $\lambda_{K}$ for the $\mathcal{A}^{(K)}$ algebras (1.1) can be thought of as a continuation of the program initiated by Zamolodchikov [1], in which one picks the chiral operator content for a proposed chiral algebra, and calculates which values of the structure constants and central charge (if any) are allowed by associativity. The non-local property of these new algebras makes the analysis more involved.

The associativity constraints on the structure constants of any chiral algebra are derived from demanding invariance of the correlation functions of the chiral currents under different ways of grouping the operators. This procedure has been formulated in terms of the braiding and fusion matrices of conformal blocks $[19,20]$. Associativity is equivalent to invariance of correlation functions under fusion transformations, while invariance under braiding transformations corresponds to demanding monodromy invariance (locality). The associativity constraints are usually enlarged to include all constraints derived from invariance of correlation functions under the group of all transformations generated by the braiding and 
fusion matrices. However, these formulations assume half-integral dimensions for the chiral algebra currents, and so must be reexamined in the case of fractional spin chiral algebras. In particular, we have found that imposing invariance of the chiral correlators under the full transformation group is too strong a condition: in general there are no solutions for the structure constants. Demanding invariance only under associativity (fusion) transformations in general fixes the structure constants completely.

To determine the associativity constraints, one would like to calculate the fourpoint function of $J^{(K)}$ currents using a Ward identity derived from the $J^{(K)} J^{(K)}$ OPE. However, for general fractional dimension of the current, $\Delta$, there are two different cuts, $(z-w)^{-2 \Delta}$ and $(z-w)^{-\Delta}$, appearing in the $J^{(K)} J^{(K)}$ OPE. This situation prevents us from performing the analytic continuation in $z$ necessary to derive a useful Ward identity. An equivalent way of describing this problem is in terms of the fusion matrix for $J^{(K)}$ four-point conformal blocks. The fusion matrix reflects the structure of cuts on the complex plane, and satisfies complicated nonlinear constraints following from conformal invariance (e.g. the pentagon identity, etc. of ref. [19]). Unfortunately, nontrivial solutions to these constraints for fields with fractional dimensions are known only in the specific cases of the $c<1$ minimal models, and the $S U(2)_{K}$ WZW theories. Therefore, to make further progress in understanding the fractional supersymmetry algebras $\mathcal{A}^{(K)}$, we find it easiest to relate them to the minimal and WZW theories.

To this end, we will briefly review the known representations of the $\mathcal{A}^{(K)}$ algebras and describe their free field realizations, as summarized in Table 1. The $\mathcal{A}^{(K)}$ algebras were first suggested $[5,6,7]$ as current algebras in the $S U(2)_{K} \otimes$ $S U(2)_{L} / S U(2)_{K+L}$ coset models. These models can, for fixed $K$, be constructed by a generalized Feigin-Fuchs procedure involving a $Z_{K}$-parafermion and a boson with background charge depending on $L$. The current $J^{(K)}$ is determined by the requirement that it commutes with the screening charges in the parafermion plus boson construction. Its dimension is found to be $\Delta=(K+4) /(K+2)$, and its explicit form in terms of $Z_{K}$-parafermion fields has been computed (see eqn. (3.7) 
below) [13]. The $L=1$ case corresponds to the unitary minimal models with $c=c_{\min }=1-6 /(K+2)(K+3)$, and $J^{(K)}$ reduces to the $\phi_{3,1}$ primary field in the BPZ [21] classification of minimal model fields. When $L$ is taken to infinity the background charge goes to zero and one recovers the $S U(2)_{K}$ WZW model. In this limit, the fractional supersymmetry current can be expressed as $J^{(K)}(z)=$ $q_{a b} J_{-1}^{a} \Phi_{(1)}^{b}(z)$, where $\Phi_{(1)}^{b}(z)$ is the adjoint representation WZW primary field of dimension $2 /(K+2), J_{-1}^{a}$ are the dimension 1 modes of the Kac-Moody currents, and $q_{a b}$ is the Killing form for $S U(2)$.

The strategy for calculating $\lambda_{K}$ is as follows. The fusion matrices have been computed for the $S U(2)_{K}$ WZW models [16] using the Wakimoto free-field realization [22]. Thus we can implement the associativity and closure conditions for the $S U(2)_{K}$ representations of $\mathcal{A}^{(K)}$ in order to determine $\lambda_{K}$ at $c=c_{S U(2)}$. Since $J^{(K)}$ is a WZW descendant of the spin-1 primary field, $\lambda_{K}$ is proportional to the $c_{111}$ chiral $S U(2)_{K}$ structure constant. The closure condition corresponds to the requirement that $c_{112}$ (the structure constant for two spin-1 fields to fuse to a spin-2 field) is zero. The generalized Feigin-Fuchs realization of the coset representations of $\mathcal{A}^{(K)}$ gives an expression for $J^{(K)}$ valid for central charges $c_{\min } \leq c \leq c_{S U(2)}$. By computing the $J^{(K)} J^{(K)}$ OPE using this expression, in which the background charge dependence is explicit, we can determine the central charge dependence of $\lambda_{K}$. Coupled with the $S U(2)_{K}$ WZW model calculation of the value of $\lambda_{K}$ at $c=c_{S U(2)}$, this determines $\lambda_{K}$ at all $c$.

Alternatively, we can determine $\lambda_{K}$ using the fusion matrices of the minimal model representations of $\mathcal{A}^{(K)}$. These have been calculated in [23,24] using the Feigin-Fuchs realization of the minimal series in terms of a free boson with background charge [25]. Here $J^{(K)}$ is identified with the $\phi_{3,1}$ Virasoro primary field. The closure condition corresponds to the decoupling of the $\phi_{5,1}$ primary field from the chiral algebra. Then the associativity argument determines $\lambda_{K}$ at the particular value of the central charge $c_{\text {min }}$, and hence provides an independent check on our calculations. 
Since most readers are more familiar with the minimal model formalism, we will present the logic of chiral algebra associativity and the calculation of $\lambda_{K}\left(c_{\min }\right)$ first, in Section 2. Section 3 then describes the determination of the central charge dependence of $\lambda_{K}$ using the generalized Feigin-Fuchs realization of $\mathcal{A}^{(K)}$, and in Section 4 we outline the technically somewhat more complicated associativity argument for the $S U(2)_{K}$ representations. We collect in appendices some old and new results for fusion and braiding matrices in the minimal and WZW models, and for $S U(2)_{K}$ Ward identities useful in calculating parafermion OPEs.

In Section 5 we discuss various issues related to the fractional supersymmetry algebras and to non-local chiral algebras in general. Since the fractional supersymmetry algebras are non-local, they obey non-trivial braiding properties. We show that the $J^{(K)}$ conformal blocks obey non-Abelian braiding properties that are closely related to the fractional spin of the current. It is interesting to ask if other non-local chiral algebras can be constructed along the lines of the $\mathcal{A}^{(K)}$ algebras presented above. In fact, the spin- $4 / 3$ parafermion algebra of [4] is closely related to the $\mathcal{A}^{(4)}$ algebra, except that it has two spin-4/3 currents instead of one. The relation between these two algebras suggests new fractional supersymmetry algebras associated with $S U(2)$. Finally, we comment on the possibility of using fields with dimensions other than those appearing in (1.1) to generate new non-local chiral algebras.

\section{Associativity Constraints on Chiral Algebras}

To compute the structure constants of a chiral operator algebra, we follow the bootstrap procedure of Belavin, Polyakov, and Zamolodchikov [21]. In particular we will express the four-point correlation functions of the theory in question in terms of the conformal blocks and then translate the associativity of the operator product algebra into conditions involving the blocks and the structure constants. In principle the blocks are known (in practice only for the minimal series and $S U(2)_{K}$ models), so the associativity condition gives constraints on the structure 
constants. We will see that for chiral algebras these constraints are sufficient to solve for the structure constants. Also, locality (monodromy invariance) of the correlation function is too strong a condition to impose: in general there are no solutions satisfying both associativity and locality.

For concreteness, we illustrate this idea in the context of the minimal unitary models, though it should be clear that the basic idea is more general. We want to calculate in the minimal unitary series with an enlarged chiral algebra. In particular, in addition to the energy-momentum tensor, suppose that the $\phi_{3,1}$ primary field is also a chiral algebra current. We take the $\phi_{3,1}$ field to be a left-moving (holomorphic) field and we consider the four-point chiral correlation function

$$
G\left(z_{i}\right)=\left\langle\phi_{3,1}\left(z_{1}\right) \phi_{3,1}\left(z_{2}\right) \phi_{3,1}\left(z_{3}\right) \phi_{3,1}\left(z_{4}\right)\right\rangle \text {. }
$$

From the minimal model fusion rules

$$
\phi_{m, 1} \times \phi_{n, 1}=\sum_{k=|m-n|+1}^{\min \{m+n-1,2 K+3-m-n\}} \phi_{k, 1}
$$

where $k$ is stepped by two in the sum, the $\phi_{3,1}$ fields satisfy the OPE

$$
\begin{aligned}
\phi_{3,1}(z) \phi_{3,1}(w)= & (z-w)^{-2 \Delta} d_{331}[1]+(z-w)^{-\Delta} d_{333}\left[\phi_{3,1}(w)\right] \\
& +(z-w)^{\Delta+1} d_{335}\left[\phi_{5,1}(w)\right] .
\end{aligned}
$$

The square brackets denote the primary field and its descendants as well as their $(z-w)$ dependence. $\Delta$ is the conformal weight of the $\phi_{3,1}$ field. The $d_{331}$ structure constant can be set to one, fixing the normalization of the $\phi_{3,1}$ field; we will do so henceforth. Note that the correlation (2.1) is symbolic of the more precise expression involving Feigin-Fuchs conjugate fields and screening charges, as described in [23]. Parameterizing the minimal model central charge by

$$
c=1-\frac{6}{(K+2)(K+3)}
$$

for $K=1,2, \ldots$, we have $\Delta=(K+4) /(K+2)$ for $K \geq 2$. Strictly speaking, the $\phi_{5,1}$ field only exists for $K \geq 4$; we will consider the cases $K=2$ and $K=3$ at the 
end of this Section. Since the $\phi_{5,1}$ fields appear in the $\phi_{3,1}$ OPE, it too must be a chiral field. Note, however, that since its dimension is $\Delta_{5,1}=3 \Delta+1$, it appears with a positive fractional power of $(z-w)$ in the $\phi_{3,1}$ OPE.

We impose associativity by requiring that $G\left(z_{i}\right)$ be the same independent of the order in which the $\phi_{3,1}$ fields are fused. For example, as $z_{1} \rightarrow z_{2}$ we can replace $\phi_{3,1}\left(z_{1}\right) \phi_{3,1}\left(z_{2}\right)$ in $G\left(z_{i}\right)$ by its OPE. Then we only have three-point functions left in the determination of $G\left(z_{i}\right)$, whose form is fixed by $\mathrm{SL}(2, \mathbf{C})$ invariance and whose normalizations are the structure constants of the chiral algebra. Choosing the normalization $d_{331}=1$, we find

$$
G\left(z_{i}\right)=F_{3}\left(z_{i}\right)+\left(d_{333}\right)^{2} F_{2}\left(z_{i}\right)+\left(d_{335}\right)^{2} F_{1}\left(z_{i}\right)
$$

where the $F_{k}\left(z_{i}\right)$ are the holomorphic conformal blocks. These blocks must be normalized so that, as $z_{1} \rightarrow z_{2}$ the expansion of the $F_{k}$ 's in powers of $z_{12}=z_{1}-z_{2}$ starts with unity, up to the $z_{i}$-dependence required by $S L(2, \mathbf{C})$ invariance. For the $\phi_{3,1}$ conformal blocks, for example, this means

$$
\begin{aligned}
& F_{1}\left(z_{i}\right)=\left(z_{12} z_{34}\right)^{\Delta+1}\left(z_{13} z_{24}\right)^{-3 \Delta-1}\left\{1+\mathcal{O}\left(z_{12}\right)\right\}, \\
& F_{2}\left(z_{i}\right)=\left(z_{12} z_{34} z_{13} z_{24}\right)^{-\Delta}\left\{1+\mathcal{O}\left(z_{12}\right)\right\}, \\
& F_{3}\left(z_{i}\right)=\left(z_{12} z_{34}\right)^{-2 \Delta}\left\{1+\mathcal{O}\left(z_{12}\right)\right\} .
\end{aligned}
$$

On the other hand, $G\left(z_{i}\right)$ could equally well have been evaluated in the limit as $z_{2} \rightarrow z_{3}$. We would have then found a new expansion in terms of conformal blocks:

$$
G\left(z_{i}\right)=F_{3}^{\prime}\left(z_{i}\right)+\left(d_{333}\right)^{2} F_{2}^{\prime}\left(z_{i}\right)+\left(d_{335}\right)^{2} F_{1}^{\prime}\left(z_{i}\right) .
$$

Since the conformal blocks form a complete basis of $S L(2, \mathbf{C})$-invariant functions for $G$, we can write

$$
F_{k}\left(z_{i}\right)=\alpha_{k j} F_{j}^{\prime}\left(z_{i}\right)
$$

for some matrix $\alpha_{k j}$ (the 'fusion matrix'). For a general four-point conformal block with fields labelled by $a, b, c$, and $d$, the fusion matrix can be represented diagrammatically as in Fig. 1. 
Denote by $A_{k}$ the vector of structure constants $A_{k}=\left\{\left(d_{335}\right)^{2},\left(d_{333}\right)^{2}, 1\right\}$ for $k=1,2,3$. Demanding that (2.5) equal (2.7) gives the condition that $A_{j}$ must be an eigenvector of the fusion matrix with eigenvalue one:

$$
A_{j} \alpha_{j k}=A_{k}
$$

In the case we are considering, $\alpha$ is a $3 \times 3$ matrix $(j, k \in\{1,2,3\})$. Expressions for the fusion matrices for the minimal unitary series are collected in Appendix A, where we find that, up to a similarity transformation that does not affect the eigenvalues,

$$
\begin{aligned}
\alpha_{j k} \sim \widetilde{\alpha}_{j k}^{(3)}(-2,-2,-2 ; \rho+1) & \\
& =\left(\begin{array}{ccc}
\frac{x^{4}}{\left(1+x^{4}\right)\left(1+x^{2}+x^{4}\right)} & \frac{-x^{2}}{1+x^{4}} & \frac{x^{2}}{1+x^{2}+x^{4}} \\
-\frac{1+x^{2}+x^{4}+x^{6}+x^{8}}{\left(1+x^{4}\right)\left(1+x^{2}+x^{4}\right)} & \frac{1-x^{2}+x^{4}}{1+x^{4}} & \frac{x^{2}}{1+x^{2}+x^{4}} \\
\frac{1+x^{2}+x^{4}+x^{6}+x^{8}}{x^{2}\left(1+x^{2}+x^{4}\right)} & 1 & \frac{x^{2}}{1+x^{2}+x^{4}}
\end{array}\right),
\end{aligned}
$$

where $\rho=\frac{1}{K+2}$ and $x=\exp (i \pi \rho)$. Since $\sum_{k} \alpha_{j k} \alpha_{k l}=\delta_{j l}$, the eigenvalues of $\alpha$ must all be \pm 1 . It is easy to check that $\alpha$ has a double eigenvalue +1 . Thus, there is a one parameter family of solutions to (2.9).

Is there some other condition on $A_{j}$ which picks out a single solution? A standard strategy is to impose locality (monodromy invariance) on the correlation functions of our theory. However, the chiral $\phi_{3,1}$ fields have fractional spins which create cuts in their OPEs. We should expect correlation functions of these fields to be multivalued, and for the locality condition to break down. We can see this explicitly by computing the 'braiding matrix' for the conformal blocks of the $\phi_{3,1}$ four-point function.

Braiding comes into play when we interchange two fields in a correlation function along some contour. The locality condition states that the resulting correlation 
function is equal to the same correlation function with the arguments in the correct radial ordering:

$$
\langle\ldots \phi(z) \psi(w) \ldots\rangle=\langle\ldots \psi(w) \phi(z) \ldots\rangle
$$

for $|w|>|z|$, where the correlator on the left hand side means the analytic continuation of the function $\langle\ldots \phi(w) \psi(z) \ldots\rangle$ along a contour that interchanges $z$ and $w$. If we perform this analytic continuation on the conformal blocks of the four-point function, we obtain a new set of blocks $\left\{F_{k}^{\prime \prime}\left(z_{i}\right)\right\}$. These blocks are linearly related to the original ones through the braiding matrix $\beta_{k j}^{+}:^{\star}$

$$
F_{k}\left(z_{i}\right)=\beta_{k j}^{+} F_{j}^{\prime \prime}\left(z_{i}\right)
$$

The action of the braiding matrix can be represented diagrammatically on fourpoint blocks as in Fig. 2.

The requirement of locality on four-point functions says that $G\left(z_{i}\right)=A_{k} F_{k}\left(z_{i}\right)=$ $A_{k} F_{k}^{\prime \prime}\left(z_{i}\right)$, which implies that $A_{j}$ is an eigenvector of $\beta^{+}$with eigenvalue one:

$$
A_{j} \beta_{j k}^{+}=A_{k}
$$

In the case we are considering (a four-point function of the $\phi_{3,1}$ field) $\beta^{+}$is a $3 \times 3$ matrix. So if it had a double eigenvalue one, then generically there would be exactly one solution of both (2.9) (associativity or fusion) and (2.13) (locality or braiding). However, in Appendix A we derive that (up to a similarity transformation)

$$
\beta_{j k}^{+} \sim \widetilde{\beta}_{j k}^{(3)+}(-2,-2,-2 ; \rho+1)=(-1)^{j+k} x^{-j^{2}-k^{2}+7 j+7 k-16} \alpha_{j k} .
$$

It is easily checked that $\beta_{j k}^{+}$does not, in general, have a double eigenvalue one, and that there is no solution to both (2.9) and (2.13). Therefore, in order to consider

$\star$ The superscript on $\beta^{+}$refers to the particular choice for the path along which the conformal blocks have been continued. This choice is reflected in the sense of the over- or undercrossing of the legs in Fig. 2. 
fractional spin chiral algebras we must drop the locality assumption. On general grounds we expect fractional spin fields in two dimensions to satisfy more complicated braiding relations than the usual Bose or Fermi statistics. The braiding matrix $\beta^{+}$in $(2.14)$ clearly shows that $\phi_{3,1}$ obeys a non-Abelian braiding relation.

We are still left with a continuous family of possible structure constants satisfying the associativity condition (2.9). In fact, this extra freedom arises because we have not fully specified our fractional supersymmetry chiral algebra. The unitary model fusion rules implied that the $\phi_{5,1}$ chiral field enters in the OPE (2.3). Thus, we should also consider the OPEs of $\phi_{5,1}$ with $\phi_{3,1}$ and with itself, typically generating yet more chiral fields.

Let us illustrate this with the $K=4$ minimal model. In this case only the $\phi_{5,1}$ field enters, and the minimal model fusion rules allow only the following couplings:

$$
\begin{aligned}
\phi_{3,1} \phi_{3,1} & \sim[1]+d_{333}\left[\phi_{3,1}\right]+d_{335}\left[\phi_{5,1}\right], \\
\phi_{3,1} \phi_{5,1} & \sim d_{335}\left[\phi_{3,1}\right] \\
\phi_{5,1} \phi_{5,1} & \sim d_{551}[1] .
\end{aligned}
$$

We choose the normalization $d_{551}=1$, if it is not zero, by changing the normalization of the $\phi_{5,1}$ field. We have already derived the associativity constraint (2.9) coming from the four-point function of $\phi_{3,1}$ fields. It turns out that the only other constraint comes from the four-point function of two $\phi_{3,1}$ fields with two $\phi_{5,1}$ fields, as illustrated in Fig. 3. This translates to

$$
d_{551}=\alpha^{\prime}\left(d_{335}\right)^{2}
$$

where $\alpha^{\prime}=\alpha_{33}^{(3)}(-2,-2,-4 ; \rho+1)$, in the notation of Appendix A. (Note that even though $\alpha_{j k}^{(3)}(-2,-2,-4)$ is generally a $3 \times 3$ matrix, when $K=4$ the fusion rules truncate it to the one element $\alpha^{\prime}$.) Using the formulas of Appendix A, it is a matter of simple algebra to determine the solution to (2.9) and (2.16) to be $d_{333}=0$ and $d_{335}=1 / \sqrt{\alpha^{\prime}}(\neq 0)$. Note, however, that there is a second solution 
to (2.9) and (2.16) in which $d_{335}=d_{551}=0$, and $d_{333}$ is non-zero. The fact that $d_{551}$ vanishes in this solution means that the $\phi_{5,1}$ field decouples entirely from the chiral algebra.

Of these two solutions, only the second one (i.e. $\left.d_{333} \neq 0, d_{335}=0\right)$ persists for larger values of $K$. For $K \geq 5$, more couplings and more fields enter into the fusion rules, but by checking a few cases it is easy to see that the number of associativity constraints increases faster than the number of couplings. Thus generically there are no solutions to the associativity constraints in which none of the fields allowed by the fusion rules decouple. (One can easily check the $K=5$ constraints explicitly to see that no coincidences reduce the number of constraints to allow such a solution.)

On the other hand, there clearly is a general solution (for all $K \geq 3$ ) in which all the fields except 1 and $\phi_{3,1}$ decouple from the chiral algebra. Thus we will define our chiral algebra to be the extended Virasoro algebra in which only the $\phi_{3,1}$ field couples. Then the only associativity constraint is (2.9) coming from the $\phi_{3,1}$ four-point function, subject to the condition $d_{335}=0$, or equivalently,

$$
A_{1}=0
$$

This is the closure condition. Because the fusion matrix $\alpha_{j k}$ in (2.9) has a double eigenvalue +1 , there is a unique solution to (2.9) subject to (2.17). Using the explicit formula (2.10) for the fusion matrix (where now we must include the similarity factors, as described in Appendix A), and the normalization of the $\phi_{3,1}$ field, $A_{3}=1$, it is a matter of straightforward algebra to deduce

$$
\left(d_{333}\right)^{2}=-\frac{4(K+6)^{2}}{3(K+4)(K+5)} \frac{\sin ^{2}(\pi \rho) \sin ^{2}(4 \pi \rho)}{\sin ^{3}(2 \pi \rho) \sin (3 \pi \rho)} \frac{\Gamma^{3}(\rho) \Gamma^{2}(4 \rho)}{\Gamma(3 \rho) \Gamma^{4}(2 \rho)},
$$

where $\rho=\frac{1}{K+2}$. This, then, is the $\phi_{3,1} \phi_{3,1}$ coupling in the chiral algebra in which the $\phi_{5,1}$ field does not appear. 
Before proceeding we should note that although (2.18) gives the correct result for $K=2$ and $K=3$, special features appear in these cases. Considering the minimal model primary fields $\phi_{n, 1}$ where $1 \leq n \leq K+1$, we see that $\phi_{5,1}$ does not exist for $K \leq 3$. Therefore, for $K=3$, there are only two conformal blocks for the $\phi_{3,1}$ four-point function and the $\alpha$ matrix (2.10) should really be a $2 \times 2$ matrix. The correct $\alpha$ matrix is easily found, however, by truncating the first row and column of (2.10). This, in essence, is what we did by imposing the closure condition $A_{1}=0(2.17)$. For $K=2$, life is even simpler. From the fusion rule (2.2), we see that the $\phi_{3,1} \phi_{3,1}$ OPE closes only on the identity. Therefore, we know a priori that $d_{333}=0$ for $K=2$. Indeed, (2.18) gives this result. Strictly speaking, the derivation given above breaks down since the $\alpha$ matrix (2.10) is illdefined: some of the entries are infinite. This is due to the degeneracies among the conformal blocks, and is a general feature whenever $K$ is not sufficiently large.

As was explained in the Introduction, the minimal model with $c=1-6 /(K+$ $2)(K+3)$ is a representation of the fractional supersymmetry chiral algebra $\mathcal{A}^{(K)}$ (1.1), with $\phi_{3,1}$ playing the role of the $J^{(K)}$ current. Thus $d_{333}$ is the structure constant for the minimal model value of the central charge, i.e. $\lambda_{K}\left(c_{\min }\right)=d_{333}(K)$.

To avoid any confusion, we should note that the value for the structure constant $d_{333}$ (2.18) derived above is not the same as that found by Dotsenko and Fateev and others $[23,24]$. The reason is that they compute the structure constants for a non-chiral theory. In particular, they consider four-point correlation functions of left-right symmetric combinations of chiral fields and demand associativity of the operator product algebra, and monodromy invariance (locality) of the correlator. For example, in the unitary model, to calculate $\widehat{d}_{333}$ they consider the four-point function $G\left(z_{i}, \bar{z}_{i}\right)=\left\langle\phi_{3,1}\left(z_{1}, \bar{z}_{1}\right) \phi_{3,1}\left(z_{2}, \bar{z}_{2}\right) \phi_{3,1}\left(z_{3}, \bar{z}_{3}\right) \phi_{3,1}\left(z_{4}, \bar{z}_{4}\right)\right\rangle$ where the $\phi_{3,1}$ OPE is

$$
\begin{aligned}
\phi_{3,1}(z, \bar{z}) \phi_{3,1}(w, \bar{w})= & |z-w|^{-4 \Delta}[1]+|z-w|^{-2 \Delta}\left|\widehat{d}_{333}\right|^{2}\left[\phi_{3,1}(w, \bar{w})\right] \\
& +|z-w|^{2 \Delta+2}\left|\widehat{d}_{335}\right|^{2}\left[\phi_{5,1}(w, \bar{w})\right] .
\end{aligned}
$$

It is crucial to note that the structure constants that appear in (2.19) are not the 
same ones that appear in the chiral OPE (2.3). Since there are no "off-diagonal" combinations of holomorphic and antiholomorphic vertex operators appearing in (2.19), it cannot be simply the product of left- and right-moving chiral OPEs. The two ways of fusing fields in $G$ give the relation between conformal blocks $\widehat{A}_{k}\left|F_{k}\left(z_{i}\right)\right|^{2}=\widehat{A}_{k}\left|F_{k}^{\prime}\left(z_{i}\right)\right|^{2}$ which in turn implies

$$
\sum_{l} \widehat{A}_{l} \alpha_{l j} \bar{\alpha}_{l k}=\widehat{A}_{j} \delta_{j k} .
$$

This determines the structure constants $\widehat{d}_{333}$ and $\widehat{d}_{335}$ uniquely. In particular, $\widehat{d}_{333} \neq d_{333}$ and $\widehat{d}_{335} \neq 0$, so it is clear that this non-chiral solution to the associativity constraints is different from the chiral solutions described above.

The fact that the fields in the non-chiral model only appear in left-right symmetric combinations ensures that once (2.20) is satisfied, so is invariance of the correlator under braiding transformations. The point is that by considering only leftright symmetric combinations, Dotsenko and Fateev restrict themselves to integer(actually zero-) spin fields, which have trivial braiding properties. We will discuss the braiding (or locality) properties of our chiral solutions in Section 5.

\section{Generalized Feigin-Fuchs Realization of $\mathcal{A}^{(K)}$}

It was noticed some time ago in ref. $[5,6,7]$ that conformal field theories with $\mathcal{A}^{(K)}(1.1)$ as their chiral algebra can be constructed by a generalized Feigin-Fuchs technique. One 'bosonizes' the $S U(2)_{K}$ WZW theory with a boson and a $Z_{K^{-}}$ parafermion, and then introduces a background charge for the boson. For special values of the background charge, unitary representations of $\mathcal{A}^{(K)}$ are found, corresponding to the coset models $S U(2)_{K} \otimes S U(2)_{L} / S U(2)_{K+L}$ with $L$ varying over the positive integers for various background charges.

In the generalized Feigin-Fuchs construction, the screening operators are $V_{+}=$ $\psi_{1} \exp \left(i \sqrt{2} \alpha_{+} \varphi\right)$ and $V_{-}=\psi_{1}^{\dagger} \exp \left(i \sqrt{2} \alpha_{-} \varphi\right)$, where $\psi_{1}$ and $\psi_{1}^{\dagger}$ are $Z_{K}$-parafermion 
currents [3]. $\varphi$ is a scalar field with background charge with

$$
\begin{gathered}
T_{\varphi}(z)=-\frac{1}{2}(\partial \varphi)^{2}+i \sqrt{2} \alpha_{0} \partial^{2} \varphi, \quad c_{\varphi}=1-24 \alpha_{0}^{2}, \\
\langle\varphi(z) \varphi(w)\rangle=-\ln (z-w), \quad \alpha_{ \pm}=\alpha_{0} \pm \sqrt{\alpha_{0}^{2}+\frac{1}{K}}
\end{gathered}
$$

The energy-momentum tensor of the $\mathcal{A}^{(K)}$ algebra is then $T=T_{\varphi}+T_{Z_{K}}$, and the total central charge is

$$
c=c_{\varphi}+c\left(Z_{K}\right)=\left(1-24 \alpha_{0}^{2}\right)+\frac{2(K-1)}{K+2} .
$$

Recall that the current $J^{(K)}(z)$ has dimension $\Delta=(K+4) /(K+2)$. Searching the $Z_{K}$-parafermion spectrum for fields of the appropriate dimension reveals only four potential candidates:

$$
\begin{gathered}
\epsilon_{1}=A_{-\frac{1}{K}}^{\dagger} \sigma_{2}, \quad \epsilon_{1}^{\dagger}=A_{-\frac{1}{K}} \sigma_{2}^{\dagger}, \\
\widehat{\epsilon}_{1}=A_{-\frac{1}{K}-1}^{\dagger} \sigma_{2}, \quad \widehat{\epsilon}_{1}^{\dagger}=A_{-\frac{1}{K}-1} \sigma_{2}^{\dagger},
\end{gathered}
$$

where $A$ and $A^{\dagger}$ are the modes of the $\psi_{1}$ and $\psi_{1}^{\dagger}$ parafermion currents and $\sigma_{2}$ and $\sigma_{2}^{\dagger} \equiv \sigma_{K-2}$ are $Z_{K}$-parafermion highest weight operators (spin fields). The definition (3.3) of the $\epsilon$-fields in terms of parafermion current modes is equivalent to the following OPE:

$$
\psi_{1}^{\dagger}(z) \sigma_{2}(w)=(z-w)^{\frac{2}{K}-1}\left\{\epsilon_{1}(w)+(z-w) \widehat{\epsilon}_{1}(w)+\ldots\right\}
$$

as well as its daggered version. The "energy" operators $\epsilon_{1}, \epsilon_{1}^{\dagger}$ have dimension $\Delta-1$ and their parafermion descendants $\widehat{\epsilon}_{1}, \widehat{\epsilon}_{1}^{\dagger}$ have dimension $\Delta$. One can show [13], using parafermion current algebra identities, that these four fields are not independent, but are related by

$$
\epsilon_{1}^{\dagger}=\epsilon_{1}, \quad \widehat{\epsilon}_{1}^{\dagger}+\widehat{\epsilon}_{1}=\left(\frac{K+2}{2}\right) \partial \epsilon_{1} .
$$

Thus there are only three independent fields of the correct dimension to form $J^{(K)}$ : $\epsilon_{1} \partial \varphi, \partial \epsilon_{1}$, and $\widehat{\epsilon}_{1}$. 
To construct $J^{(K)}$ we search for the combination of the above fields that commutes with the screening charges $V_{ \pm}[5,6,7]$. Using the $\psi_{1}, \psi_{1}^{\dagger}$ OPEs with $\epsilon_{1}, \widehat{\epsilon}_{1}$ :

$$
\begin{aligned}
& \psi_{1}(z) \epsilon_{1}(w)=\left(\frac{2}{K}\right) \frac{\sigma_{2}(w)}{(z-w)}+\left(\frac{K+2}{K}\right) \partial \sigma_{2}(w)+\ldots \\
& \psi_{1}(z) \widehat{\epsilon}_{1}(w)=\left(\frac{K^{2}+2 K-4}{K^{2}}\right) \frac{\sigma_{2}(w)}{(z-w)^{2}}-\left(\frac{2 K+4}{K^{2}}\right) \frac{\partial \sigma_{2}(w)}{(z-w)}+\ldots
\end{aligned}
$$

as well as the daggered versions of these relations, the condition that $J^{(K)}$ commutes with $V_{ \pm}$determines the relative normalizations of the possible terms $[13,26]$

$$
J^{(K)}(z)=\sqrt{\frac{K \Delta}{4 c}}\left\{\left[\sqrt{2} \epsilon_{1} \partial \varphi-i \alpha_{0}(K+2) \partial \epsilon_{1}\right]+\frac{i K\left(\alpha_{+}-\alpha_{-}\right)}{K+4}\left[\widehat{\epsilon}_{1}-\widehat{\epsilon}_{1}^{\dagger}\right]\right\},
$$

where the overall normalization has been chosen to match (1.1). The terms in square brackets are Virasoro primary combinations.

Now that we have an explicit expression for $J^{(K)}(z)$, it is possible to calculate the chiral algebra $\left(\mathcal{A}^{(K)}\right)$ that it satisfies. Because $(3.7)$ is Virasoro primary, the part of $\mathcal{A}^{(K)}$ involving the energy-momentum tensor is standard:

$$
\begin{aligned}
T(z) T(w) & =\frac{c}{2}(z-w)^{-4}\left\{1+\frac{4}{c}(z-w)^{2} T(w)+\ldots\right\} \\
T(z) J^{(K)}(w) & =\Delta(z-w)^{-2}\left\{J^{(K)}(w)+\frac{1}{\Delta}(z-w) \partial J^{(K)}(w)+\ldots\right\},
\end{aligned}
$$

where the dots denote the Virasoro descendants appearing with higher integer powers of $(z-w)$. The $J^{(K)} J^{(K)}$ OPE, of the form

$$
\begin{aligned}
J^{(K)}(z) J^{(K)}(w)= & (z-w)^{-2 \Delta}\left\{1+\frac{2 \Delta}{c}(z-w)^{2} T(w)+\ldots\right\} \\
& +\lambda_{K}(c)(z-w)^{-\Delta}\left\{J^{(K)}(w)+\frac{1}{2}(z-w) \partial J^{(K)}(w)+\ldots\right\}+\ldots
\end{aligned}
$$

would at first sight seem to be easy to calculate: all we have to do is take the OPE of (3.7) with itself. However, when we do that we find that $\lambda_{K}(c)$ is only 
determined in terms of the $S U(2)_{K}$ structure constant $c_{111}$, which governs the fusing of two chiral spin-1 primary fields to give a spin-1 field.

To compute the OPE of $J^{(K)}(z)$ with itself as it is given in (3.7), we need the leading terms of the OPEs of the parafermion fields $\epsilon_{1}(z)$ and $\eta(z) \equiv \widehat{\epsilon}_{1}(z)-\widehat{\epsilon}_{1}^{\dagger}(z)$. (Note that when $K=2, \widehat{\epsilon}_{1}(z)=\widehat{\epsilon}_{1}^{\dagger}(z)$, so $\eta(z)$ is absent.) In Appendix B we calculate these OPEs using the definition of the $Z_{K}$-parafermion theory in terms of the $S U(2)_{K}$ WZW theory, plus the $S U(2)_{K}$ Ward identities. The results for the leading terms of interest to us are

$$
\begin{aligned}
\epsilon_{1}(z) \epsilon_{1}(0) & =-c_{110} \frac{2}{K} z^{-2 \Delta+2}+c_{111} \frac{2 \sqrt{K}}{(K-2)(K+4)} z^{-\Delta+2} \eta(0)+\ldots \\
\eta(z) \eta(0) & =-c_{110} \frac{2(K-2)(K+4)}{K^{2}} z^{-2 \Delta}-c_{111} \frac{3(K-4)}{\sqrt{K}(K-2)} z^{-\Delta} \eta(0)+\ldots \\
\epsilon_{1}(z) \eta(0) & =\eta(z) \epsilon_{1}(0)=c_{110} \mathcal{O}\left(z^{-2 \Delta+2}\right)+c_{111} \frac{2}{\sqrt{K}} z^{-\Delta} \epsilon_{1}(0)+\ldots
\end{aligned}
$$

Here $c_{110}$ is the $S U(2)_{K}$ structure constant for two $S U(2)$ spin-1 primaries to fuse to the identity. It can be set to one, fixing the normalization of the spin-1 fields; we will do so henceforth. $c_{111}$ is the chiral $S U(2)_{K}$ structure constant mentioned before. By $\mathcal{O}\left(z^{-2 \Delta+2}\right)$ in the last equation, we mean only that the leading term proportional to $c_{110} z^{-2 \Delta+1}$ vanishes.

Using these OPEs and the explicit expression (3.7) for $J^{(K)}$, we can calculate the leading terms in the $J^{(K)} J^{(K)}$ OPE. In particular, the coefficient of the identity in (3.9) is determined by the $c_{110}$ pieces in the $\left(\epsilon_{1} \partial \varphi\right)\left(\epsilon_{1} \partial \varphi\right), \partial \epsilon_{1} \partial \epsilon_{1}$, and $\eta \eta$ OPEs. Potential contributions from $\partial \epsilon_{1} \eta$ and $\eta \partial \epsilon_{1}$ OPEs do not contribute since the $c_{110}$ term of the $\epsilon_{1} \eta \mathrm{OPE}$ is of order $\mathcal{O}\left(z^{-2 \Delta+2}\right)$. The normalization of $J^{(K)}$ in (3.7) was actually determined by demanding that the $J^{(K)} J^{(K)}$ OPE close on the identity with coefficient one. The coefficient $\lambda_{K}$ of $J^{(K)}$ in (3.9) can be extracted by computing the coefficient of the $\epsilon_{1} \partial \varphi$ terms. The $c_{111}$ pieces of the $\epsilon_{1} \eta, \eta \epsilon_{1}, \epsilon_{1} \partial \epsilon_{1}$ and $\partial \epsilon_{1} \epsilon_{1}$ OPEs contribute, giving

$$
\lambda_{K}^{2}(c)=\frac{2 K^{2}\left(c_{111}\right)^{2}}{3(K+4)(K+2)}\left[\frac{3(K+4)^{2}}{K(K+2)} \frac{1}{c}-1\right]
$$


where we have used the relation between the central charge and background charge (3.2). This argument evaluates $\lambda_{K}$ by computing only the coefficient of the $\epsilon_{1} \partial \varphi$ term in $J^{(K)}$. A similar computation using the $c_{111}$ pieces of the $\epsilon_{1} \epsilon_{1}$ and $\eta \eta$ OPEs shows that the $J^{(K)} J^{(K)}$ OPE indeed has the form (3.9), closing precisely on $J^{(K)}$. Other terms, not included in (3.10), indicate that one other chiral primary field, proportional to parafermion descendants of the $\sigma_{4}$ spin field, may enter in the $J^{(K)} J^{(K)}$ OPE. The structure constant for this additional field is proportional to $c_{112}$, the chiral $S U(2)_{K}$ structure constant for two spin-1 fields to fuse to a spin-2 field. Since we have defined the fractional supersymmetry algebras to be those in which no fields other than $T(z)$ and $J^{(K)}(z)$ (and their Virasoro descendants) appear, we must demand that $c_{112}=0$. This is the closure condition, discussed in the last section. In the next section we will show that it is compatible with the associativity constraints of the chiral $S U(2)_{K}$ WZW model.

If we fix the background charge in (3.2) so that the central charge takes on the minimal unitary model values $c_{\min }=1-6 /(K+2)(K+3)$ we know from the discussion in the previous section that $\lambda_{K}\left(c_{\min }\right)=d_{333}$ (where we have chosen to normalize the $\phi_{3,1}$ field in the same way that $J^{(K)}$ is normalized, i.e. $d_{331}=1$ ). Comparing to (3.11) gives a relation between the structure constants:

$$
\left(d_{333}\right)^{2}=\frac{4}{3}\left(c_{111}\right)^{2} \frac{(K+6)^{2}}{(K+4)(K+5)} .
$$

Using the value for $d_{333}$ (2.18) computed by associativity and closure, we obtain the explicit expression for $c_{111}$ (1.3) given in the Introduction.

A priori the $S U(2)_{K}$ structure constant $c_{111}$ could depend on the central charge, thereby invalidating the argument leading to (3.12). Indeed, $c_{111}$ should be thought of as a structure constant in the coset model representation of $\mathcal{A}^{(K)}$, and therefore it should be determined by requiring associativity in that model. However, because of the simple way in which the Feigin-Fuchs boson $\varphi$ enters into the expression (3.7) for $J^{(K)}, c_{111}$ can be identified as the structure constant determined by associativity of the chiral $S U(2)_{K}$ theory. In particular, correlators 
involving $J^{(K)}$ can be broken up into sums of products of $Z_{K}$-parafermion correlators and Feigin-Fuchs boson correlators. Since the latter only involve the boson "current" $\partial \varphi$, they are independent of the background charge $\alpha_{0}$, and the chiral $Z_{K}$-parafermion correlators must satisfy associativity by themselves. We will show in the next section that, just as in the minimal model case examined in the last section, there is only one solution to the associativity and closure conditions for the chiral $S U(2)_{K}$ WZW model and thus only one for the chiral $Z_{K}$-parafermion model since it is simply the WZW model stripped of a free boson. Therefore, for given $K, c_{111}$ should be independent of the central charge of the generalized Feigin-Fuchs realization of $\mathcal{A}^{(K)}$.

\section{Associativity Constraints in Chiral $S U(2)_{K}$ WZW Models}

Setting the background charge to zero in the generalized Feigin-Fuchs representation of the last section corresponds to taking the $L \rightarrow \infty$ limit of the $S U(2)_{K} \otimes S U(2)_{L} / S U(2)_{K+L}$ coset model. In this limit we expect to recover the $S U(2)_{K}$ WZW theory. Now, the $S U(2)_{K}$ WZW model has been solved [16] by using the Wakimoto representation [22] to represent the spin- $j$ primary fields $\Phi_{m}^{(j)}$ in terms of free fields with background charge, allowing the derivation of integral expressions for the conformal blocks. It is found [16] that the fusion matrices of the $S U(2)_{K}$ spin- $j$ primary fields are the same up to similarity transformations as those of the $\phi_{2 j+1,1}$ primaries of the $K$ th minimal unitary model. The similarity transformations depend on the normalization constants of the conformal blocks, but do not affect the eigenvalues of the fusion matrices. This means that the analysis of associativity constraints presented for the minimal model in Section 2 works through in precisely the same way for the WZW theory since it only depends on the number of +1 eigenvalues of the fusion matrix. In particular, we are still assured of a solution to the associativity conditions if we demand that only the energy momentum tensor and the spin-1 field (corresponding to $\phi_{3,1}$ ) appear, all other fields decoupling from our chiral algebra. In this case $c_{111}$ is determined by the analog of eqn. (2.9), 
where $\alpha_{j k}$ is now the $S U(2)_{K}$ fusion matrix and $A_{k}=\left\{\frac{1}{6}\left(c_{112}\right)^{2}, \frac{1}{2}\left(c_{111}\right)^{2}, 1\right\}$ is the vector of $S U(2)_{K}$ structure constants. The factors of $\frac{1}{6}$ and $\frac{1}{2}$ in the vector $A_{k}$ are standard $S U(2)$ group factors explained in Appendix B, (B12)-(B13). The closure condition, corresponding to $(2.17)$, is $c_{112}=0$, and tells us that the spin-2 fields decouple. Using the results for the normalization constants for the $S U(2)_{K}$ conformal blocks [16], summarized in Appendix C, we find the expression (1.3) for $c_{111}$ given in the Introduction.

It is important to note that this way of determining $c_{111}$ is independent of the argument used in the last section, where it was fixed by comparing to the results of the minimal model associativity argument of Section 2. The fact that both give the same result is a nontrivial check of the calculation of $\lambda_{K}(c)$ using the generalized Feigin-Fuchs representation of Section 3.

We have made the vague identification of the WZW spin-1 primary with the $J^{(K)}$ current in the last two paragraphs. Actually, $J^{(K)}$ is a Kac-Moody descendant of the WZW spin-1 primary field. To see this, take $\alpha_{0}=0$ in the generalized FeiginFuchs model of the previous section. The limit of (3.7) gives the fractional current in terms of parafermion fields and a free boson:

$$
J^{(K)}(z)=\sqrt{\frac{K+4}{6}}\left\{\epsilon_{1}(z) \partial \varphi(z)+\frac{i \sqrt{2 K}}{(K+4)} \eta(z)\right\} .
$$

Using the standard connection between $Z_{K}$-parafermions plus a free boson and the $S U(2)_{K}$ WZW theory (reviewed in Appendix B) we can reexpress (4.1) as

$$
J^{(K)}(z)=\frac{i}{\sqrt{3(K+4)}}\left(J_{-1}^{-} \Phi_{+}^{(1)}(z)-\sqrt{2} J_{-1}^{0} \Phi_{0}^{(1)}(z)-J_{-1}^{+} \Phi_{-}^{(1)}(z)\right)
$$

where $J_{-1}^{a}$ are Kac-Moody current modes and $\Phi_{m}^{(1)}(z)$ is the WZW spin-1 primary field with dimension $2 /(K+2)$. (This expression is proportional to $q_{a b} J_{-1}^{a} \Phi_{(1)}^{b}$, the form for $J^{(K)}$ mentioned in Table 1.) 
The associativity condition we are really interested in involves the $J^{(K)}$ current, and not the spin-1 fields. To fix the $J^{(K)} J^{(K)}$ structure constant in this theory requires knowledge of the fusion matrix for the four-point function of $J^{(K)}$ currents. It is not obvious, a priori, that this fusion matrix is the same as that for the spin-1 fields derived in [16] (and summarized in Appendix C). However, it turns out that the fusion matrix for the $J^{(K)}$ field differs from that of the spin-1 field only by a similarity transformation. This is in line with the observation that the fusion matrix reflects the structure of cuts in the OPEs of the chiral fields which in turn depends only on the fractional part of the dimensions of those fields. Since the dimension of $J^{(K)}$ differs from that of the WZW spin-1 field by an integer (one), we expect the fusion matrix to be the same up to a similarity transformation. Of course, the actual solution for the structure constants depends on that similarity transformation.

In the remainder of this section, we will outline the calculation of the $J^{(K)}$ four-point conformal block fusion matrix in the chiral $S U(2)_{K} \mathrm{WZW}$ model. We will show that it implies a value for $\lambda_{K}$ in agreement with the one found by our previous arguments.

We follow ref. [16] in which Dotsenko uses the Wakimoto representation [22] to solve the $S U(2)_{K}$ WZW model. We introduce a scalar field with background charge $\alpha_{0}$ and a $c=2$ system, $\omega$ and $\omega^{+}$, of dimension 0 and 1 respectively, satisfying

$$
\left\langle\omega(z) \omega^{+}(w)\right\rangle=-\left\langle\omega^{+}(z) \omega(w)\right\rangle=\frac{i}{z-w}
$$

The combined boson plus $\left(\omega, \omega^{+}\right)$system gives a realization of the $S U(2)_{K}$ WZW model. Since the total central charge of this system is $c=3-24 \alpha_{0}^{2}$, the level must be given by $K+2=1 / 4 \alpha_{0}^{2}$. The screening momenta are $\alpha_{+}=2 \alpha_{0}$ and $\alpha_{-}=0$. The screening charge $Q_{+}=\oint d z \omega^{+}(z) V_{\alpha_{+}}(z)$, where $V_{\alpha}(z)=\exp \{i \sqrt{2} \alpha \varphi(z)\}$, 
commutes with the Kac-Moody currents given by [22]

$$
\begin{aligned}
& J^{+}=\omega^{+}, \\
& i J^{0}=\omega \omega^{+}+\frac{1}{2 \sqrt{2} \alpha_{0}} \partial \varphi, \\
& J^{-}=\omega^{2} \omega^{+}+i K \partial \omega+\frac{1}{\sqrt{2} \alpha_{0}} \omega \partial \varphi .
\end{aligned}
$$

The spin- $j$ primary fields are expressed in terms of binomial coefficients and the free fields as (in the normalization of Appendix B)

$$
\Phi_{m}^{(j)}(z)=i^{j-m}\left(\begin{array}{c}
2 j \\
j+m
\end{array}\right)^{1 / 2}(\omega(z))^{j-m} V_{\alpha_{j}}(z),
$$

where $\alpha_{j}=-2 \alpha_{0} j$.

From these formulas we can immediately write down the expression for $J^{(K)}$ in terms of free fields

$$
J^{(K)}=-\sqrt{\frac{K+4}{3}} \partial \omega V_{-\alpha_{+}}
$$

In order to evaluate the $J^{(K)}$ four-point correlation function we must construct the Feigin-Fuchs conjugate current $\widetilde{J}^{(K)}$. A field and its Feigin-Fuchs conjugate have the same conformal and $S U(2)_{K}$ properties; both are necessary in order to cancel the background charges and construct non-zero correlation functions in the Wakimoto representation. The conjugate to the identity operator is found to be [16]

$$
\widetilde{1}=\left(\omega^{+}\right)^{-K-1} V_{-\alpha_{+}(K+1)} .
$$

This implies the neutrality conditions for correlation functions, $N^{-}-N^{+}=K+1$ and $\sum \alpha_{i}=-\alpha_{+}(K+1)$, where $N^{+}$and $N^{-}$are the number of $\omega^{+}$and $\omega$ zeromodes, respectively, and the $\alpha_{i}$ are the "momenta" of the $\varphi$ zero-modes entering in the correlator. The construction of the fields conjugate to the spin- $j$ primaries, 
$\widetilde{\Phi}_{m}^{(j)}$, is outlined in [16]. Using the spin-1 conjugates and (4.2) it is straightforward to construct the conjugate of $J^{(K)}$ :

$$
\begin{aligned}
\widetilde{J}^{(K)}= & -\sqrt{\frac{K+4}{3}}\left\{(\partial \omega)\left(\omega^{+}\right)-\frac{i K\left(K^{2}-1\right)}{2(K+4)}\left(\omega^{+}\right)^{-1}\left(\partial^{2} \omega^{+}\right)\right. \\
& +\frac{i K\left(K^{2}-1\right)}{2}\left(\omega^{+}\right)^{-2}\left(\partial \omega^{+}\right)^{2}-\frac{K(K-1)(2 K+5)}{2 \sqrt{2} \alpha_{0}(K+4)}(\partial \varphi)\left(\omega^{+}\right)^{-1}\left(\partial \omega^{+}\right) \\
& \left.+\frac{(K-1)(K-2)}{2 \sqrt{2} \alpha_{0}(K+4)}\left(\partial^{2} \varphi\right)-\frac{i\left(K^{2}-1\right)(K+2)}{(K+4)}(\partial \varphi)^{2}\right\}\left(\omega^{+}\right)^{-K} V_{-K \alpha_{+}}
\end{aligned}
$$

We can now write the $J^{(K)}$ four-point correlator as

$$
G_{J}\left(z_{i}\right)=\left\langle J^{(K)}\left(z_{1}\right) J^{(K)}\left(z_{2}\right) J^{(K)}\left(z_{3}\right) \widetilde{J}^{(K)}\left(z_{4}\right) Q_{+} Q_{+}\right\rangle
$$

where the two insertions of the screening charge $Q_{+}$are necessary to satisfy the neutrality conditions mentioned above. Using the explicit expressions derived above for $J^{(K)}, \widetilde{J}^{(K)}$, and $Q_{+}, G_{J}\left(z_{i}\right)$ can be written as a sum of products of $\omega, \omega^{+}$and $\varphi$ free-field correlators (modulo the zero-mode insertions required to satisfy the neutrality constraints). However, the contours for the integrals in the definition of the screening charges has not been specified in (4.9). As in the usual FeiginFuchs procedure [23], there are only three linearly independent choices of contours for two screening charges in a four-point function. Thus, evaluating the free-field correlation functions we can write $G_{J}\left(z_{i}\right)$ in terms of three conformal blocks

$$
G_{J}\left(z_{i}\right)=\left(z_{13} z_{24}\right)^{-2 \Delta}[\eta(1-\eta)]^{\frac{2}{K+2}} \sum_{k=1}^{3} A_{k}\left(N_{k}\right)^{-1} I_{k}(\eta),
$$

where $z_{i j}=z_{i}-z_{j}$ and $\eta$ is the projective invariant $\eta=z_{12} z_{34} / z_{13} z_{24}$. The $A_{k}$ are the as yet undetermined coefficients of the blocks, and the $N_{k}$ 's are normalization constants. The integral expressions for the conformal blocks have the following 
form

$$
\left.\begin{array}{l}
I_{1}(\eta)=\int_{1}^{\infty} d u_{1} \int_{1}^{u_{1}} d u_{2} \\
I_{2}(\eta)=\int_{1}^{\infty} d u_{1} \int_{0}^{\eta} d u_{2} \\
I_{3}(\eta)=\int_{0}^{\eta} d u_{1} \int_{0}^{u_{1}} d u_{2}
\end{array}\right\}\left[u_{1} u_{2}\left(u_{1}-\eta\right)\left(u_{2}-\eta\right)\left(u_{1}-1\right)\left(u_{2}-1\right)\right]^{-2 \rho}\left(u_{1}-u_{2}\right)^{2 \rho} R\left(u_{1}, u_{2}, \eta\right),
$$

where

$$
\begin{aligned}
R\left(u_{1}, u_{2}, \eta\right)= & \frac{1}{u_{1}^{2}\left(u_{2}-\eta\right)^{2}}+\frac{1}{u_{1}^{2}\left(u_{2}-1\right)^{2}}+\frac{1}{\left(u_{1}-\eta\right)^{2} u_{2}^{2}} \\
& +\frac{1}{\left(u_{1}-\eta\right)^{2}\left(u_{2}-1\right)^{2}}+\frac{1}{\left(u_{1}-1\right)^{2} u_{2}^{2}}+\frac{1}{\left(u_{1}-1\right)^{2}\left(u_{2}-\eta\right)^{2}}
\end{aligned}
$$

and $\rho=1 /(K+2)$. The normalization constant $N_{k}$ is defined as the coefficient of the leading term in $\eta$ of $I_{k}(\eta)$ as $\eta \rightarrow 0$. Using the results in [23] we find that

$$
\begin{aligned}
& N_{1}=\left[\frac{-10 \pi^{2}(K+2)^{2}(K+4)}{3(K+5)(K+6)}\right]\left[\frac{1}{\sin (\pi \rho) \sin (2 \pi \rho)}\right]\left[\frac{\Gamma(5 \rho)}{\Gamma^{2}(\rho) \Gamma(3 \rho)}\right] \\
& N_{2}=\left[\frac{-16 \pi^{2}(K+2)^{2}}{(K+4)^{2}}\right]\left[\frac{\sin (4 \pi \rho)}{\sin ^{3}(2 \pi \rho)}\right]\left[\frac{\Gamma^{2}(4 \rho)}{\Gamma^{4}(2 \rho)}\right] \\
& N_{3}=\left[\frac{-3 \pi^{2}(K+2)^{2}}{(K+4)}\right]\left[\frac{\sin (3 \pi \rho)}{\sin ^{2}(\pi \rho) \sin (2 \pi \rho)}\right]\left[\frac{\Gamma(3 \rho)}{\Gamma^{3}(\rho)}\right] .
\end{aligned}
$$

As $z_{1} \rightarrow z_{2}$, we find that $G_{J}\left(z_{i}\right)$ has the leading order behavior,

$$
\begin{aligned}
G_{J}\left(z_{i}\right)= & A_{3}\left(z_{12} z_{34}\right)^{-2 \Delta}\left\{1+\mathcal{O}\left(z_{12}\right)\right\}+A_{2}\left(z_{12} z_{34} z_{13} z_{24}\right)^{-\Delta}\left\{1+\mathcal{O}\left(z_{12}\right)\right\} \\
& +A_{1}\left(z_{12} z_{34}\right)^{\Delta-1}\left(z_{13} z_{24}\right)^{-3 \Delta+1}\left\{1+\mathcal{O}\left(z_{12}\right)\right\} .
\end{aligned}
$$

We are now able to make the identification, as in Section 2 , of the $A_{i}$ 's with the structure constants of the $\mathcal{A}^{(K)}$ chiral algebra. In particular if we set $A_{3}=1$ to properly normalize the $J^{(K)}$ current, then $A_{2}=\lambda_{K}^{2}$. The first term of $(4.14)$ implies that a field of dimension $3 \Delta-1$ enters into the $J^{(K)} J^{(K)}$ OPE (whereas the dimension of the $\phi_{5,1}$ field that entered into the $\phi_{3,1} \phi_{3,1}$ OPE in Section 2 was $3 \Delta+1)$. In fact, the dimension $3 \Delta-1$ field corresponds to a level 2 Kac-Moody descendant of the spin-2 primary field of dimension $3(\Delta-1)$. 
The $I_{k}$ integrals are related under the change of variables $\eta \rightarrow 1-\eta$ by the matrix $\widetilde{\alpha}^{J}$ :

$$
I_{j}(\eta)=\widetilde{\alpha}_{j k}^{J} I_{k}(1-\eta)
$$

We calculate $\widetilde{\alpha}^{J}$ using the contour deformation techniques described in Appendix A. Briefly, we make the change of variables $\eta \rightarrow 1-\eta$ and $u_{i} \rightarrow 1-u_{i}$ in the $I_{k}$ integrals (4.11) which changes the limits of integration but leaves the integrand invariant. We can then pull the contours of integration back to their original positions and obtain (4.15). In actuality we do not need to do all of this work, since the rational function $R$ is merely a spectator in all of these manipulations; its effects are manifest only in the normalization constants $N_{k}$. The rest of the integrand is of the same form as the integrands that appear in the minimal model conformal blocks (A4). Therefore, in the notation of Appendix A (A11), we find

$$
\widetilde{\alpha}_{j k}^{J}=\widetilde{\alpha}_{j k}^{(3)}(-2,-2,-2 ; \rho)
$$

This is the matrix that appeared (2.9) in our discussion of the minimal model associativity condition in Section 2. Since $\rho=\widetilde{\rho}-1$ we have that $x=\exp (i \pi \rho)=$ $-\widetilde{x}$, but since $(2.9)$ is even in $x$, there is no difference between the two matrices.

Examining (4.10) we see that the fusion matrix for the conformal blocks of the $J^{(K)}$ four-point functions is given by $\left(N_{j}\right)^{-1} \widetilde{\alpha}_{j k}^{J}\left(N_{k}\right)$. We can now determine $\lambda_{K}$ by requiring that the $A_{k}$ 's satisfy the associativity condition, given by the eigenvalue equation

$$
\sum_{j} A_{j}\left(N_{j}\right)^{-1} \widetilde{\alpha}_{j k}^{J}\left(N_{k}\right)=A_{k}
$$

and by imposing the closure condition $A_{1}=0$. This gives the result

$$
\lambda_{K}^{2}\left(c_{S U(2)}\right)=\frac{16}{3(K+4)} \frac{\sin ^{2}(\pi \rho) \sin ^{2}(4 \pi \rho)}{\sin ^{3}(2 \pi \rho) \sin (3 \pi \rho)} \frac{\Gamma^{3}(\rho) \Gamma^{2}(4 \rho)}{\Gamma(3 \rho) \Gamma^{4}(2 \rho)}
$$

in agreement with (1.2) and (1.3). 


\section{Discussion}

In this section we briefly comment on a few interesting questions associated with the fractional supersymmetry chiral algebras $\mathcal{A}^{(K)}$, and with fractional spin chiral algebras in general. (a) We will first describe the braiding properties of the fractional supersymmetry currents $J^{(K)}$. (b) Next, we investigate the possible existence of new chiral algebras differing from the fractional supersymmetry chiral algebras by having two or more $J^{(K)}$ currents, although still with the same dimensions as in the $\mathcal{A}^{(K)}$ algebra. (c) Finally, we address the possibility of constructing in our framework non-local chiral algebras with a fractional spin current of dimension different from that of $J^{(K)}$.

a. It was shown in Section 2 that the fractional spin current $J^{(K)}$ does not satisfy simple Bose or Fermi statistics. Instead, the correlation functions of these currents transform according to some more complicated representation of the braid group, given by the braiding matrices $\beta_{j k}^{ \pm}$introduced earlier. We can examine the properties of the four-point conformal block braiding matrices by relating them to the fusion matrix and "twisting" matrices $\gamma^{ \pm}[19,20]$. The latter are just the diagonal matrices of phases of conformal blocks as two operators which are "contracted" are exchanged. More concretely, consider two fields $\phi_{1}\left(z_{1}\right)$ and $\phi_{2}\left(z_{2}\right)$ in a block which is normalized as $z_{1} \rightarrow z_{2}$. The phases under interchange of these two fields are simply determined by the powers of $\left(z_{1}-z_{2}\right)$ appearing in their OPE: if this OPE contains a field $\phi_{i}\left(z_{2}\right)$ on the right hand side, then the corresponding monodromy is $\exp \left\{ \pm i \pi\left(\Delta_{i}-\Delta_{1}-\Delta_{2}\right)\right\}$. So, in general we have

$$
\gamma_{j}^{ \pm}(a, b)=\mathrm{e}^{ \pm i \pi\left(\Delta_{j}-\Delta_{a}-\Delta_{b}\right)} .
$$

The relations between $\alpha, \beta^{ \pm}$, and $\gamma^{ \pm}$that we want are most easily described diagrammatically. We adopt the notation shown in Fig. 4 for the action of $\gamma$ on conformal blocks (as well as the notations for $\alpha$ and $\beta$ introduced earlier in Figs. 1 and 2), where the sense of the crossing of lines indicates which way the 
corresponding blocks are analytically continued in the corresponding variables. Then Fig. 5 illustrates the relation

$$
\beta_{i j}^{ \pm}(a, b, c, d)=\alpha_{i k}(a, b, c, d) \gamma_{k}^{ \pm}(b, c) \alpha_{k j}(c, b, d, a) .
$$

A similar and simpler argument gives

$$
\delta_{i j}=\alpha_{i k}(a, b, c, d) \alpha_{k j}(b, c, d, a)
$$

Since we are interested in the braiding properties of the fractional supersymmetry current, we will take all external legs to be the $J^{(K)}$ field, of dimension $\Delta=$ $(K+4) /(K+2)$. The $\gamma_{j}^{ \pm}$are then given by

$$
\gamma^{ \pm}=\left\{\exp \left( \pm \frac{2 \pi i}{K+2}\right),-\exp \left(\mp \frac{2 \pi i}{K+2}\right), \exp \left(\mp \frac{4 \pi i}{K+2}\right)\right\}
$$

where the last element, $\gamma_{3}^{ \pm}$, corresponds to two $J^{(K)}$ currents fusing to the identity, the middle to $J^{(K)}$, and the first to the extra chiral field (called $\phi_{5,1}$ in Section 1) that should decouple by the closure condition. We can drop the external leg arguments of $\alpha, \beta^{ \pm}$, and $\gamma^{ \pm}$, and write, as a simple consequence of (5.2) and (5.3)

$$
\left(\beta^{ \pm}\right)^{n}=\alpha\left(\gamma^{ \pm}\right)^{n} \alpha
$$

where $\gamma^{ \pm}$is to be thought of as a diagonal matrix, and matrix multiplication is understood. Since correlation functions of $J^{(K)}$ currents are invariant under $\alpha$ transformations (this is the associativity condition), they will also be invariant under $\left(\beta^{ \pm}\right)^{n}$ by (5.5) if $n$ is such that $\left(\gamma^{ \pm}\right)^{n}=1$. From (5.4) we see that if $K$ is even this is achieved for $n=K+2$, and if $K$ is odd, for $n=2(K+2)$.

However, from the discussion of Section 1 we recall that the $J^{(K)}$ correlation functions satisfy not only the associativity condition but also the closure condition which states that no other fields than $T$ and $J^{(K)}$ enter into the $\mathcal{A}^{(K)}$ chiral algebra. 
In particular, the $\phi_{5,1}$ field decouples, so the top row and first column of the $\alpha$, $\beta^{ \pm}$, and $\gamma^{ \pm}$matrices play no role. This means that the $J^{(K)}$ four-point function will be invariant under $\left(\beta^{ \pm}\right)^{n}$ transformations for $n$ such that the lower two entries in $\left(\gamma^{ \pm}\right)^{n}$ are one. This permits the stronger statement that, for the case where $K=4 m$ for some integer $m$, the $J^{(K)}$ correlator is invariant under $\left(\beta^{ \pm}\right)^{n}$ with $n=(K+2) / 2$. In this case $n$ is odd, so this is a condition involving braidings in which the $J^{(K)}$ fields are interchanged an odd number of times, and so can be thought of as a non-trivial generalization of the notion of statistics for the $J^{(K)}$ currents. It is important to note that these statistics are not just the Abelian phases of "fractional statistics." Indeed, under a single interchange of two $J^{(K)}$ fields, we obtain two new fields related to the old $J^{(K)}$ fields not by an overall phase, but by the action of the matrix $\beta^{ \pm}$on their chiral vertices.

In fact, using the explicit form for $\beta^{ \pm}$derived in Appendix A, one can show that demanding invariance of the $J^{(K)}$ correlator when $K=4 m$ under $\left(\beta^{ \pm}\right)^{(K+2) / 2}$ transformations, as well as under fusion $(\alpha)$ transformations, is equivalent to demanding the closure condition. This suggests that the algebras with $K=4 m$ can be characterized as those that are invariant under the subgroup of the full transformation group generated by $\alpha$ and $\left(\beta^{ \pm}\right)^{(K+2) / 2}$. We do not know whether a similar characterization exists for the $K \neq 4 m$ theories.

b. One reason for considering extended Virasoro algebras like $\mathcal{A}^{(K)}$ is that infinite numbers of Virasoro primaries are organized into extended chiral algebra descendants of a smaller number of chiral algebra primary fields. For this reason it is important to look for extended algebras which are in some sense maximal. So far, we have considered the case where the Virasoro algebra is extended by only one additional current $J^{(K)}$. For the spin-4/3 $(K=4)$ case, Fateev and Zamolodchikov [4] have constructed an extended algebra with two additional currents of the same dimension $\Delta=(K+4) /(K+2)$ : 


$$
\begin{aligned}
\psi(z) \psi(w) & =\sqrt{2} \lambda_{4}(z-w)^{-\Delta}\left\{\psi^{\dagger}(w)+\ldots\right\}, \\
\psi^{\dagger}(z) \psi^{\dagger}(w) & =\sqrt{2} \lambda_{4}(z-w)^{-\Delta}\{\psi(w)+\ldots\}, \\
\psi(z) \psi^{\dagger}(w) & =(z-w)^{-2 \Delta}\left\{1+\frac{2 \Delta}{c}(z-w)^{2} T(w)+\ldots\right\} .
\end{aligned}
$$

Here $\lambda_{4}$ is given by (1.2) and (1.3) with $K=4$, implying that $\left(c_{111}\right)^{2}=1$ and

$$
\lambda_{4}^{2}=\frac{2}{9}\left[\frac{8}{c}-1\right] .
$$

Note that $J^{(4)}=\left(\psi+\psi^{\dagger}\right) / \sqrt{2}$ satisfies the $\mathcal{A}^{(4)}$ algebra (1.1). An important property of this splitting of the $\mathcal{A}^{(4)}$ algebra is that there is only a single cut appearing on the right hand side of the OPEs (5.6). This implies that the $\psi(z)$ and $\psi^{\dagger}(z)$ fields obey Abelian braid relations, and simplifies the analysis of the spin- $4 / 3$ algebra considerably.

The existence of this splitting of the $\mathcal{A}^{(4)}$ algebra might have been guessed on other grounds. Let us consider the minimal model with $c=6 / 7(K=4)$, the tricritical three-state Potts model [27], which we have seen is a representation of the $\mathcal{A}^{(4)}$ chiral algebra. Its Virasoro primary fields may be labelled by two indices $\phi_{m, n}$. When we enlarge the Virasoro symmetry to the $\mathcal{A}^{(4)}=\{J, T\}$ or the $\left\{\psi, \psi^{\dagger}, T\right\}$ extended algebra, some of the $\phi_{m, n}$ become descendants with respect to the extended algebra of other Virasoro primary fields. Specifically, $\phi_{3,1}$ becomes a descendant of the identity $\phi_{1,1}$. In the fractional supersymmetry algebra (1.1), there is one $\phi_{3,1}$ field: $\phi_{3,1}=J_{-\Delta} \phi_{1,1}$. In Fateev and Zamolodchikov's spin$4 / 3$ algebra, there are two $\phi_{3,1}$ fields: $\psi_{-\Delta} \phi_{1,1}$ and $\psi_{-\Delta}^{\dagger} \phi_{1,1}$. Let us denote the characters corresponding to the Virasoro primaries $\phi_{m, n}$ by $\chi_{m, n}$. Then the spin$4 / 3$ extended algebra characters are given by

$$
\operatorname{ch}_{n}=\chi_{1, n}+2 \chi_{3, n}+\chi_{5, n} .
$$

The resulting modular invariant partition function $\mathcal{Z}\left(D_{4}\right)$ corresponds to the $\left(D_{4}, A_{6}\right)$ case (or the $D$ case) in the $A D E$ classification [28]. This argument easily gener- 
alizes to all the $S U(2)_{4} \otimes S U(2)_{L} / S U(2)_{4+L}$ coset models (where the Virasoro characters are generalized to the appropriate branching functions [29]).

This picture and the $A D E$ classification of modular invariant partition functions [28] suggests that when $K=4 m$ there exist new symmetry algebras differing from the fractional supersymmetries $\mathcal{A}^{(K)}$ by having additional fractional spin currents entering into the chiral algebra, similar to (5.6). To simplify the analysis, the goal is to enlarge these algebras so that they will satisfy Abelian braiding relations.

c. The associativity analysis for extended Virasoro algebras in the minimal models can be applied to currents other than the $\phi_{3,1}$ field, which was the one considered in Section 2. For simplicity, let us consider chiral algebras involving the energymomentum tensor and just one other current, $\phi_{M, 1}$. From the minimal model fusion rules, the fusion matrix, $\alpha_{M}$, for the four-point function of this current is an $M \times M$ matrix. By $(5.3),\left(\alpha_{M}\right)^{2}=1$, so all its eigenvalues are \pm 1 . Recall that the associativity condition on our chiral algebra states that the vector of structure constants of the algebra is an eigenvector with eigenvalue +1 of $\alpha_{M}$. Let $n$ denote the number of +1 eigenvalues. Then there will be an $n-1$ dimensional space of solutions to the associativity conditions. (One entry of the vector of structure constants is fixed by the normalization of the $\phi_{M, 1}$ field.)

However, for our proposed chiral algebra to have only $T$ and $\phi_{M, 1}$ as chiral currents, there must be more constraints to prevent other fields from coupling. These are just the closure conditions for the chiral algebra. In particular, the $\phi_{M, 1} \phi_{M, 1}$ OPE closes on the set $\left\{\phi_{1,1}, \phi_{3,1}, \ldots, \phi_{2 M-1,1}\right\}$, so there are $M-2$ (if $M$ is odd) or $M-1$ (if $M$ is even) further conditions on the vector of structure constants needed to decouple the unwanted fields. This means that, generically, for there to be a solution to the associativity and closure conditions, we must have $n \geq M-1$ (for $M$ odd) or $n=M$ (for $M$ even). If $n=M$, then $\alpha_{M}$ would be the identity matrix, which is not the case. In fact, using the result for the fusion matrix given in Appendix A (A11), we find that for $M=2 m$ the number of +1 eigenvalues is $m$, while for $M=2 m+1$ the number of +1 eigenvalues is $m+1$. 
Thus the only generic solution to the associativity and closure conditions occurs for $M=3$, which correspond to the fractional supersymmetry chiral algebras $\mathcal{A}^{(K)}$ we have been studying in this paper.

Though the above argument was presented for the minimal models, it only depended on the eigenvalues of the fusion matrix. It should be clear from the discussions of previous sections that this argument is therefore valid for the whole range of $S U(2)_{K} \otimes S U(2)_{L} / S U(2)_{K+L}$ coset representations.

This work was supported in part by the National Science Foundation.

\section{APPENDIX A}

This Appendix presents explicit formulas for the fusion and braiding matrices for conformal blocks of the $c<1$ minimal models. These matrices, or at least parts of them, have been calculated in different ways in ref. [23,24]. We will follow Dotsenko and Fateev's method in what follows; for more details, the reader is referred to ref. [23].

The unitary minimal models have central charge given in (2.4), and primary fields ${ }^{\star} \phi_{n, m}$ where $m, n$ are integers satisfying $1 \leq n \leq K+1,1 \leq m \leq n$. We specialize to the subalgebra with $m=1$, since that is all we use in the rest of this paper. The conformal dimension of the $\phi_{n, 1}$ field is $\Delta_{n, 1}=\frac{1}{4}(n-1)[n-1+(n+$ 1) $/(K+2)]$.

Dotsenko and Fateev derive integral expressions for the conformal blocks of four-point functions using a Feigin-Fuchs [25] construction of the minimal models as a boson with background charge. We omit the details of introducing conjugate fields and screening charges in what follows. They show that there are $n$

\footnotetext{
$\star$ We have used the opposite convention of [23] so that our $\phi_{n, m}$ is their $\phi_{m, n}$.
} 
independent conformal blocks, where $n=\left(n_{1}+n_{2}+n_{3}-n_{4}\right) / 2$, so that

$$
\begin{aligned}
G\left(z_{i}\right) & =\left\langle\phi_{n_{1}, 1}\left(z_{1}\right) \phi_{n_{2}, 1}\left(z_{2}\right) \phi_{n_{3}, 1}\left(z_{3}\right) \phi_{n_{4}, 1}\left(z_{4}\right)\right\rangle \\
& =\sum_{k=1}^{n} A_{k}^{(n)}(a, b, c ; \widetilde{\rho}) F_{k}^{(n)}\left(a, b, c ; \widetilde{\rho} ; z_{i}\right),
\end{aligned}
$$

where we have defined the useful parameters

$$
\begin{aligned}
& a=1-n_{1}, \quad b=1-n_{2}, \\
& c=1-n_{3}, \quad \widetilde{\rho}=1+\frac{1}{K+2} .
\end{aligned}
$$

Without loss of generality we have taken $n_{4} \geq n_{1}, n_{2}, n_{3}$. The $A_{k}^{(n)}(a, b, c ; \widetilde{\rho})$ in (A.1) are the structure constants to be determined by the associativity conditions explained in Section 2. The expression for the correctly normalized conformal blocks is

$$
\begin{aligned}
F_{k}^{(n)}\left(a, b, c ; \widetilde{\rho} ; z_{i}\right)= & z_{13}^{-\Delta_{1}-\Delta_{2}-\Delta_{3}+\Delta_{4}} z_{14}^{-\Delta_{1}+\Delta_{2}+\Delta_{3}-\Delta_{4}} z_{34}^{\Delta_{1}+\Delta_{2}-\Delta_{3}-\Delta_{4}} z_{24}^{-2 \Delta_{2}} \\
& \times \eta^{a b \tilde{\rho} / 2}(1-\eta)^{b c \tilde{\rho} / 2}\left\{N_{k}^{(n)}(a, b, c ; \widetilde{\rho})\right\}^{-1} I_{k}^{(n)}(a, b, c ; \widetilde{\rho} ; \eta),
\end{aligned}
$$

where we have defined the integrals

$$
\begin{aligned}
I_{k}^{(n)}(a, b, c ; \widetilde{\rho} ; \eta)= & \int_{1}^{\infty} d u_{1} \ldots \int_{1}^{u_{n-k-1}} d u_{n-k} \int_{0}^{\eta} d u_{n-k+1} \ldots \int_{0}^{u_{n-2}} d u_{n-1} \prod_{i<j}^{n-1}\left(u_{i}-u_{j}\right)^{2 \widetilde{\rho}} \\
& \times \prod_{i=1}^{n-k} u_{i}^{a \tilde{\rho}}\left(u_{i}-\eta\right)^{b \widetilde{\rho}}\left(u_{i}-1\right)^{c \widetilde{\rho}} \prod_{i=n-k+1}^{n-1} u_{i}^{a \widetilde{\rho}}\left(\eta-u_{i}\right)^{b \widetilde{\rho}}\left(1-u_{i}\right)^{c \widetilde{\rho}},
\end{aligned}
$$

and the following normalization constants [23]

$$
\begin{aligned}
N_{k}^{(n)}(a, b, c ; \widetilde{\rho})= & \prod_{i=1}^{n-k} \frac{\Gamma(i \widetilde{\rho})}{\Gamma(\widetilde{\rho})} \prod_{i=1}^{k-1} \frac{\Gamma(i \widetilde{\rho})}{\Gamma(\widetilde{\rho})} \prod_{i=0}^{k-2} \frac{\Gamma(1+\widetilde{\rho}(a+i)) \Gamma(1+\widetilde{\rho}(b+i))}{\Gamma(2+\widetilde{\rho}(a+b+k-2+i))} \\
& \times \prod_{i=0}^{n-k-1} \frac{\Gamma(-1-\widetilde{\rho}(a+b+c+2 n-4-i)) \Gamma(1+\widetilde{\rho}(c+i))}{\Gamma(-\widetilde{\rho}(a+b+2 k-2+i))} .
\end{aligned}
$$

We use the notations $\Delta_{i}=\Delta_{n_{i}, 1}, z_{i}-z_{j}=z_{i j}, \eta=\left(z_{12} z_{34}\right) /\left(z_{13} z_{24}\right)$, and $\eta$ is taken to lie on the real axis between 0 and 1 . Other values of $\eta$ can be reached 
by analytic continuation. These formulas describe conformal blocks which are correctly normalized for the field at $z_{1}$ fusing with the field at $z_{2}$. This follows from the fact that in the $z_{1} \rightarrow z_{2}(\eta \rightarrow 0)$ limit

$$
I_{k}^{(n)}(a, b, c ; \widetilde{\rho} ; \eta) \rightarrow N_{k}^{(n)}(a, b, c ; \widetilde{\rho}) \eta^{(k-1)(1+\widetilde{\rho}(a+b+k-2))} f(\eta)
$$

where $f$ is some analytic function of $\eta$ which satisfies $f(0)=1$.

The fusion matrix $\alpha$ relates the above conformal blocks to those which are appropriately normalized as $z_{2} \rightarrow z_{3}(\eta \rightarrow 1)$. To calculate the relation between these two sets of blocks, change variables in $I_{k}^{(n)}$ to $\widetilde{\eta}=1-\eta$ and $\widetilde{u}_{i}=1-u_{i}$, to find

$$
\begin{aligned}
I_{k}^{(n)}(a, b, c ; \widetilde{\rho} ; \eta)= & \int_{-\infty}^{0} d \widetilde{u}_{1} \ldots \int_{\widetilde{u}_{n-k-1}}^{0} d \widetilde{u}_{n-k} \int_{\widetilde{\eta}}^{1} d \widetilde{u}_{n-k+1} \ldots \int_{\widetilde{u}_{n-2}}^{1} d \widetilde{u}_{n-1} \prod_{i<j}^{n-1}\left(\widetilde{u}_{j}-\widetilde{u}_{i}\right)^{2 \widetilde{\rho}} \\
& \times \prod_{i=1}^{n-k}\left(-\widetilde{u}_{i}\right)^{c \widetilde{\rho}}\left(\widetilde{\eta}-\widetilde{u}_{i}\right)^{b \widetilde{\rho}}\left(1-\widetilde{u}_{i}\right)^{a \widetilde{\rho}} \prod_{i=n-k+1}^{n-1} \widetilde{u}_{i}^{c \widetilde{\rho}}\left(\widetilde{u}_{i}-\widetilde{\eta}\right)^{b \widetilde{\rho}}\left(1-\widetilde{u}_{i}\right)^{a \widetilde{\rho}}
\end{aligned}
$$

Notice that the integrands of (A.7) and (A.4) are the same except for the switch of $a$ and $c$; the difference between the two expressions is the limits of integration. In the complex plane, however, the only singularities are at $0, \eta, 1$ and $\infty$ so that using contour deformation the integration limits in (A.7) can be pulled over to match those in (A.4). In this way we can calculate the elements of the transformation matrix $\widetilde{\alpha}$, defined by

$$
I_{k}^{(n)}(a, b, c ; \widetilde{\rho} ; \eta)=\widetilde{\alpha}_{k j}^{(n)}(a, b, c ; \widetilde{\rho}) I_{j}^{(n)}(c, b, a ; \widetilde{\rho} ; \widetilde{\eta}=1-\eta) .
$$

The $I_{j}^{(n)}$ 's on the right hand side are now in the correct form for conformal blocks in the $\widetilde{\eta} \rightarrow 0$ basis. From the complete expression (A.3) for the conformal blocks and the normalization of the $I_{k}^{(n)}$,s (A.6), we see that the fusion matrix $\alpha$ is given 
by

$$
\alpha_{k j}^{(n)}(a, b, c ; \widetilde{\rho})=\left\{N_{k}^{(n)}(a, b, c ; \widetilde{\rho})\right\}^{-1} \widetilde{\alpha}_{k j}^{(n)}(a, b, c ; \widetilde{\rho}) N_{j}^{(n)}(c, b, a ; \widetilde{\rho})
$$

Note that if $a=c, \alpha$ differs from $\widetilde{\alpha}$ by a similarity transformation and so has the same eigenvalues.

In ref. [23] Dotsenko and Fateev calculate one column of $\widetilde{\alpha}$ using the above described contour manipulation techniques. We find that it is not difficult to calculate the entire matrix using the same approach. If we define the auxiliary variable $\widetilde{x}=e^{i \pi \tilde{\rho}}$, the function $[d]=\widetilde{x}^{d}-\widetilde{x}^{-d}$, and for integral $d$ and $d^{\prime}$ the symbol

$$
\left[\begin{array}{l}
d^{\prime} \\
d
\end{array}\right]=\left\{\begin{array}{cc}
\prod_{i=d}^{d^{\prime}}[i] & d^{\prime} \geq d \\
1 & d^{\prime}<d
\end{array}\right.
$$

then the explicit form for the $\widetilde{\alpha}$ matrix is

$$
\begin{aligned}
\widetilde{\alpha}_{k j}^{(n)}(a, b, c ; \widetilde{\rho})= & \sum_{i=\max (0, k+j-n-1)}^{\min (k-1, j-1)}(-1)^{k+j-i}[b+c+2 j-3][b+c+n-k-1+2 i] \\
\times & {\left[\begin{array}{c}
a+n-j-1 \\
a+k-1-i
\end{array}\right]\left[\begin{array}{c}
b+j-2 \\
b+i
\end{array}\right]\left[\begin{array}{c}
c+n-k-1+i \\
c+n-k
\end{array}\right] } \\
& \times\left[\begin{array}{c}
a+b+c+n+k-4 \\
a+b+c+n-2+i
\end{array}\right]\left[\begin{array}{c}
j-1 \\
1+i
\end{array}\right]\left[\begin{array}{c}
n-j \\
k-i
\end{array}\right] \\
& \times\left\{\left[\begin{array}{c}
b+c+n-2+i \\
b+c+n-k-1+i
\end{array}\right]\left[\begin{array}{c}
b+c+n-k+j-2+i \\
b+c+j-2+i
\end{array}\right]\right. \\
& \left.\times\left[\begin{array}{c}
j-1-i \\
1
\end{array}\right]\left[\begin{array}{c}
n-k-j+1+i \\
1
\end{array}\right]\right\}^{-1} .
\end{aligned}
$$

Now we would like to consider the braiding matrix $\beta$ for four-point blocks. This matrix relates blocks properly normalized as $z_{1} \rightarrow z_{2}$ to those normalized as $z_{1} \rightarrow z_{3}$. Since the operators are radially ordered, for $z_{1}$ to approach $z_{3}$ it must 
be continued around the operator at $z_{2}$. In terms of the integral representations of the conformal blocks, this corresponds to continuing the parameter $\eta \rightarrow 1 / \eta$. Different transformations result depending on whether we continue above or below the singularity at $\eta=1$ in the complex plane. This analytic continuation can be performed explicitly using expressions (A.3)-(A.5) for the conformal blocks.

However, it turns out $[19,20]$ that the braiding matrices are simply expressible in terms of the fusion matrix and "twisting" matrices $\gamma^{ \pm}$, introduced above in Section 5. The relation we are interested in between $\alpha, \beta^{ \pm}$, and $\gamma^{ \pm}$is most easily described diagrammatically. We adopt the notation for $\alpha, \beta$, and $\gamma$ introduced earlier in Figs. 1, 2, and 4, where the sense of the crossing of lines indicates which way the corresponding blocks are analytically continued in the corresponding variables. Then Fig. 6 illustrates the relation

$$
\beta_{j k}^{ \pm}(a, b, c, d)=\gamma_{j}^{\mp}(a, b) \alpha_{j k}(b, a, c, d) \gamma_{d}^{ \pm}(k, b) .
$$

Note that the equivalence of (A.12) with the alternate expression for $\beta^{ \pm}$in terms of $\alpha$ and $\gamma^{ \pm}$given by (5.2) is just an expression of the Yang-Baxter relation that braiding matrices must satisfy. Translating into the parameters used above to describe the minimal model conformal blocks, we derive from (A.12) the simple formula for the braiding matrix in terms of the fusion matrix

$$
\begin{aligned}
\beta_{j k}^{(n) \pm}(a, b, c ; \widetilde{\rho})= & \mathrm{e}^{\mp i \pi\left(\Delta_{1}+\Delta_{2}+\Delta_{3}-\Delta_{4}+j+k-2\right)} \alpha_{j k}^{(n)}(b, a, c ; \widetilde{\rho}) \\
& \times \widetilde{x}^{ \pm(1-j)(a+b+j-2)} \widetilde{x}^{ \pm(1-k)(a+c+k-2)} .
\end{aligned}
$$




\section{APPENDIX B}

This Appendix describes the calculation of the OPEs of the $Z_{K}$-parafermion fields $\epsilon_{1}$ and $\eta$, introduced in Section 3. These fields are parafermion descendants of the $\sigma_{2}$ spin field. Since the $Z_{K}$-parafermion theory is simply the $S U(2)_{K} / U(1)$ coset theory, our strategy will be to express these fields in terms of $S U(2)_{K}$ WZW fields and currents, and use the $S U(2)_{K}$ Ward identities to calculate their OPEs. Since the $\sigma_{2}$ spin field is related to the WZW spin-1 primary field [3], we will concentrate on the WZW spin-1 with WZW spin-1 OPE.

The singular terms in the $S U(2)_{K}$ current-current OPE are

$$
\begin{aligned}
J^{+}(z) J^{-}(w) & =\frac{K}{(z-w)^{2}}+\frac{2 J^{0}(w)}{(z-w)} \\
J^{0}(z) J^{ \pm}(w) & = \pm \frac{J^{ \pm}(w)}{(z-w)} \\
J^{0}(z) J^{0}(w) & =\frac{K / 2}{(z-w)^{2}}
\end{aligned}
$$

The spin- $j$ primaries $\Phi_{m}^{(j)}$ have dimension $\Delta_{j}=j(j+1) /(K+2)$. We choose their relative normalizations so that

$$
\begin{aligned}
J_{0}^{0} \Phi_{m}^{(j)} & =m \Phi_{m}^{(j)}, \\
J_{0}^{ \pm} \Phi_{m}^{(j)} & =\sqrt{(j \pm m+1)(j \mp m)} \Phi_{m \pm 1}^{(j)},
\end{aligned}
$$

where $J_{0}^{ \pm, 0}$ are the zero modes of the Kac-Moody currents. We fix the overall normalization of the spin-1 fields by

$$
\Phi_{+}^{(1)}(z) \Phi_{-}^{(1)}(0)=1 \cdot z^{-4 /(K+2)}+\ldots
$$

If the $Z_{K}$-parafermion currents $\psi_{1}$ and $\psi_{1}^{+}$are defined by

$$
\begin{aligned}
J^{+} & =\sqrt{K} \psi_{1} \exp \{i \varphi \sqrt{2 / K}\} \\
J^{0} & =i \sqrt{K / 2} \partial \varphi \\
J^{-} & =\sqrt{K} \psi_{1}^{+} \exp \{-i \varphi \sqrt{2 / K}\}
\end{aligned}
$$

where $\varphi$ is a free boson satisfying $\langle\varphi(z) \varphi(w)\rangle=-\ln (z-w)$, then the parafermion 
fields are related to $\Phi_{m}^{(1)}$ by [3]

$$
\begin{aligned}
& \Phi_{+}^{(1)}=\sigma_{2} \exp \{i \varphi \sqrt{2 / K}\} \\
& \Phi_{0}^{(1)}=\sqrt{K / 2} \epsilon_{1} \\
& \Phi_{-}^{(1)}=\sigma_{2}^{+} \exp \{-i \varphi \sqrt{2 / K}\}
\end{aligned}
$$

The coefficients in (B.5) are determined by (B.2)-(B.4), the definition of $\epsilon_{1}(3.3)$, and the choice of normalization of the spin fields:

$$
\sigma_{2}(z) \sigma_{2}^{+}(w)=1 \cdot z^{-\frac{2(K-2)}{K(K+2)}}+\ldots
$$

From (B.4) and (B.5), and the definition of $\eta=\widehat{\epsilon}_{1}-\widehat{\epsilon}_{1}^{+}$, it is also easy to see that

$$
\eta=\frac{1}{\sqrt{K}}\left(J_{-1}^{-} \Phi_{+}^{(1)}+\frac{4 \sqrt{2}}{K} J_{-1}^{0} \Phi_{0}^{(1)}-J_{-1}^{+} \Phi_{-}^{(1)}\right) .
$$

Following ref. [15], introduce $S U(2)$-invariant combinations depending on a new auxiliary parameter $x$

$$
\begin{gathered}
J(x, z)=J^{-}(z)+2 x J^{0}(z)-x^{2} J^{+}(z), \\
\Phi^{(j)}(x, z)=\sum_{m=-j}^{j}\left(\begin{array}{c}
2 j \\
j+m
\end{array}\right)^{1 / 2} x^{j+m} \Phi_{m}^{(j)}(z),
\end{gathered}
$$

from which it follows with our normalization (B.2) that

$$
\begin{aligned}
& J_{0}^{+}(x) \Phi^{(j)}(x, z)=\partial_{x} \Phi^{(j)}(x, z) \\
& J_{0}^{0}(x) \Phi^{(j)}(x, z)=-j \Phi^{(j)}(x, z) \\
& J_{0}^{-}(x) \Phi^{(j)}(x, z)=0 .
\end{aligned}
$$

We have introduced the deformed current modes $J_{m}^{\alpha}(x)$ which obey the usual KacMoody current mode commutation relations. They are defined by

$$
\begin{aligned}
J_{n}^{+}(x) & =J_{n}^{+} \\
J_{n}^{0}(x) & =J_{n}^{0}-x J_{n}^{+} \\
J_{n}^{-}(x) & =J_{n}^{-}+2 x J_{n}^{0}-x^{2} J_{n}^{+} .
\end{aligned}
$$

In terms of these fields and modes, the spin- $j_{1}$ with spin- $j_{2}$ OPE can be written in 
a unified way as

$$
\begin{aligned}
\Phi^{\left(j_{1}\right)}(x, z) \Phi^{\left(j_{2}\right)}(y, 0)= & \sum_{j_{3}} c_{j_{1} j_{2} j_{3}} z^{\Delta_{j_{3}}-\Delta_{j_{1}}-\Delta_{j_{2}}} \sum_{m=0}^{\infty} z^{m} \sum_{\left\{\alpha_{i}\right\}} J_{-1}^{\alpha_{1}}(y) \ldots J_{-1}^{\alpha_{m}}(y) \\
& \times \sum_{k, l} C_{k, l}^{\left\{\alpha_{i}\right\}}(x-y)^{k+j_{1}+j_{2}-j_{3}}\left(J_{0}^{+}(y)\right)^{l} \Phi^{\left(j_{3}\right)}(y, 0) .
\end{aligned}
$$

The sum over $j_{3}$ runs over the spins allowed by the fusion rules, and the normalization is set by $C_{0,0}=1$. The structure constants $c_{j_{1} j_{2} j_{3}}$ can be related to three-point functions by [15]

$$
\begin{aligned}
\left\langle\Phi_{m_{1}}^{\left(j_{1}\right)}\left(z_{1}\right) \Phi_{m_{2}}^{\left(j_{2}\right)}\left(z_{2}\right) \Phi_{m_{3}}^{\left(j_{3}\right)}\left(z_{3}\right)\right\rangle= & {\left[j_{1} j_{2} j_{3}\right]\left(\begin{array}{ccc}
j_{1} & j_{2} & j_{3} \\
m_{1} & m_{2} & m_{3}
\end{array}\right) c_{j_{1} j_{2} j_{3}} } \\
& \times z_{12}^{\Delta_{j_{3}}-\Delta_{j_{1}}-\Delta_{j_{2}}} z_{13}^{\Delta_{j_{2}}-\Delta_{j_{1}}-\Delta_{j_{3}}} z_{23}^{\Delta_{j_{1}}-\Delta_{j_{2}}-\Delta_{j_{3}}}
\end{aligned}
$$

where

$$
\left[j_{1} j_{2} j_{3}\right]=\sqrt{\frac{\left(j_{1}+j_{2}-j_{3}\right) !\left(j_{1}-j_{2}+j_{3}\right) !\left(-j_{1}+j_{2}+j_{3}\right) !\left(j_{1}+j_{2}+j_{3}+1\right) !}{\left(2 j_{1}\right) !\left(2 j_{2}\right) !\left(2 j_{3}\right) !}}
$$

and the second term in (B.12) is the standard 3-j symbol [30].

The coefficients $C_{k, l}^{\left\{\alpha_{i}\right\}}$ in (B.11) can be determined recursively using the KacMoody Ward identities. In particular, by acting on both sides of (B.11) with $J_{n}^{a}(y, 0)$ for $n=0,1$, we obtain the relations, where $\left[\Phi^{1} \Phi^{2}\right]$ denotes the right hand side of (B.11),

$$
\begin{aligned}
& J_{0}^{+}(y, 0)\left[\Phi^{1} \Phi^{2}\right]=\left(\partial_{x}+\partial_{y}\right)\left[\Phi^{1} \Phi^{2}\right] \\
& J_{0}^{0}(y, 0)\left[\Phi^{1} \Phi^{2}\right]=\left((x-y) \partial_{x}-j_{1}-j_{2}\right)\left[\Phi^{1} \Phi^{2}\right] \\
& J_{0}^{-}(y, 0)\left[\Phi^{1} \Phi^{2}\right]=(x-y)\left(2 j_{1}-(x-y) \partial_{x}\right)\left[\Phi^{1} \Phi^{2}\right] \\
& J_{1}^{+}(y, 0)\left[\Phi^{1} \Phi^{2}\right]=z \partial_{x}\left[\Phi^{1} \Phi^{2}\right] \\
& J_{1}^{0}(y, 0)\left[\Phi^{1} \Phi^{2}\right]=z\left((x-y) \partial_{x}-j_{1}\right)\left[\Phi^{1} \Phi^{2}\right] \\
& J_{1}^{-}(y, 0)\left[\Phi^{1} \Phi^{2}\right]=z(x-y)\left(2 j_{1}-(x-y) \partial_{x}\right)\left[\Phi^{1} \Phi^{2}\right] .
\end{aligned}
$$


These are sufficient to solve for the $C_{k, l}^{\left\{\alpha_{i}\right\}}$ using the current algebra (B.1). The result we find for the leading terms in the $j_{1}=j_{2}=1 \mathrm{OPE}$ is

$$
\begin{aligned}
\Phi^{(1)}(x, z) \Phi^{(1)}(y, 0)= & c_{110} z^{-2 \Delta_{1}}(x-y)^{2}\left\{1+\frac{2 z}{K}\left[J_{-1}^{0}(y)+(x-y)^{-1} J_{-1}^{-}(y)\right]+\mathcal{O}\left(z^{2}\right)\right\} \\
& +c_{111} z^{-\Delta_{1}}(x-y)\left\{\left[\frac{(x-y)}{2} J_{0}^{+}(y)+1\right] \Phi^{(1)}(y, 0)+\mathcal{O}(z)\right\} \\
& +c_{112} z^{\Delta_{1}}\{\mathcal{O}(1)\} .
\end{aligned}
$$

Expanding according to (B.8), we see that the normalization adopted for the WZW spin-1 fields (B.3) corresponds to setting $c_{110}=1$.

All the information we need to compute the leading terms of the $\epsilon_{1}$ and $\eta$ OPEs is now at hand. By (B.5), (B.7), and (B.9) we can express the $\epsilon_{1}$ field in terms of the WZW spin-1 primary as

$$
\epsilon_{1}=\sqrt{\frac{2}{K}} \Phi_{0}^{(1)}=\left.\frac{1}{\sqrt{K}}\left[\partial_{y} \Phi^{(1)}(y, 0)\right]\right|_{y=0}=\left.\frac{1}{\sqrt{K}}\left[J_{0}^{+}(y) \Phi^{(1)}(y, 0)\right]\right|_{y=0} .
$$

We use this expression in (B.15) by taking $x$ - and $y$-derivatives of both sides, then evaluating at $x=y=0$. The leading term proportional to $c_{111}$ vanishes, and the term proportional to $c_{110}$ contributes

$$
\epsilon_{1}(z) \epsilon_{1}(0)=-c_{110}\left(\frac{2}{K}\right) z^{-2 \Delta+2}+c_{111} \mathcal{O}\left(z^{-\Delta+2}\right)+\ldots
$$

where $\Delta=\Delta_{1}+1=(K+4) /(K+2)$. The $\epsilon_{1} \eta$ OPE is somewhat harder to evaluate. From (B.7) we see that

$$
\eta=\left.\frac{1}{2 \sqrt{K}}\left(\partial_{y}^{2}+\frac{4}{K} \partial_{w} \partial_{y}+\partial_{w}^{2}\right)\left[J_{-1}(w, 0) \Phi^{(1)}(y, 0)\right]\right|_{x=y=0}
$$


A simple contour deformation argument using (B.16) and (B.18) shows that

$$
\begin{aligned}
\epsilon_{1}(z) \eta(0)= & \frac{1}{2 K} \partial_{x}\left(\partial_{y}^{2}+\frac{4}{K} \partial_{w} \partial_{y}+\partial_{w}^{2}\right) \\
& \times\left.\left[J_{-1}(w)+\frac{1}{z}\left(2(w-x)+(w-x)^{2} \partial_{x}\right)\right]\left(\Phi^{(1)}(x, z) \Phi^{(1)}(y, 0)\right)\right|_{x=y=w=0} \\
= & \left.\frac{1}{K}\left[\frac{1}{z}\left(\partial_{x}^{2}-\partial_{y}^{2}\right)+\mathcal{O}(1)\right]\left(\Phi^{(1)}(x, z) \Phi^{(1)}(y, 0)\right)\right|_{x=y=0}
\end{aligned}
$$

In the second step we evaluated the $w$-derivatives, set $w=0$, and kept only the leading power of $z$. The leading term proportional to $c_{110}$ in (B.15) vanishes, and the term proportional to $c_{111}$ contributes

$$
\epsilon_{1}(z) \eta(0)=c_{110} \mathcal{O}\left(z^{-2 \Delta+2}\right)+\frac{2 c_{111}}{\sqrt{K}} z^{-\Delta} \epsilon_{1}(0)+\ldots
$$

Finally, the $\eta(z) \eta(0)$ OPE is evaluated in a similar manner, using (B.18) and a somewhat more complicated contour manipulation, to obtain the leading $c_{110}$ term

$$
\eta(z) \eta(0)=-c_{110}\left(\frac{2(K+4)(K-2)}{K^{2}}\right) z^{-2 \Delta}+\ldots
$$

The formulas (B.17), (B.20), and (B.21) include only the minimum number of terms in the $Z_{K}$-parafermion OPEs needed in Section 3 to evaluate the $\lambda_{K}$ coupling constant in the $J^{(K)} J^{(K)}$ OPE. There are other contributions to these OPEs which we have not calculated in this Appendix. Thus, for example, the dots in (B.21) denote not only parafermion descendants of the identity, but also parafermion descendants of the $\sigma_{2}$ spin field (proportional to $c_{111}$ ) and the $\sigma_{4}$ spin field (proportional to $c_{112}$ ). It is a straightforward, if laborious, exercise to calculate these terms along the lines indicated above; in fact, we have included the leading $c_{111}$ terms of the $\epsilon_{1} \epsilon_{1}$ and $\eta \eta$ OPEs in (3.10). 


\section{APPENDIX C}

In this Appendix we assemble the integral expressions for the conformal blocks of correlators of spin- $j$ primary fields of the $S U(2)_{K}$ WZW model, as calculated in ref. [16]. The Wakimoto representation used to calculate these conformal blocks is outlined in Section 4.

The spin- $j$ primary fields, $\Phi_{m}^{(j)}$, of the $S U(2)_{K}$ WZW model have dimensions $\Delta_{j}=j(j+1) /(K+2)$. Since the $m$-dependence of the $\Phi_{m}^{(j)}$ OPEs is known from $S U(2)_{K}$ Ward identities (B.12), it is sufficient to consider correlation functions of fields with $m= \pm j$. In [16] correlators of two spin- $j$ with two spin- $l$ fields are considered. For $j>l$, it is shown that there are $n$ independent conformal blocks, where $n=2 l+1$. Thus

$$
\begin{aligned}
G_{j l}\left(z_{i}\right) & =\left\langle\Phi_{-j}^{(j)}\left(z_{1}\right) \Phi_{l}^{(l)}\left(z_{2}\right) \Phi_{-l}^{(l)}\left(z_{3}\right) \Phi_{j}^{(j)}\left(z_{4}\right)\right\rangle \\
& =\sum_{k=1}^{n} A_{k}^{(n)}(a, b, c ; \rho) F_{k}^{(n)}\left(a, b, c ; \rho ; z_{i}\right),
\end{aligned}
$$

where we have defined the parameters

$$
a=-2 j, \quad b=c=-2 l, \quad \rho=\frac{1}{K+2}
$$

The expression for the correctly normalized conformal blocks is

$$
\begin{aligned}
F_{k}^{(n)}(a, b, c ; \rho ; \eta)= & \left(z_{13} z_{24}\right)^{-2 \Delta_{l}} z_{14}^{2 \Delta_{l}-2 \Delta_{j}} \eta^{a b \rho / 2}(1-\eta)^{b c \rho / 2} \\
& \times\left\{N_{k}^{(n)}(R: a, b, c ; \rho)\right\}^{-1} I_{k}^{(n)}(R: a, b, c ; \rho ; \eta),
\end{aligned}
$$

where we have defined the integrals

$$
\begin{aligned}
I_{k}^{(n)}(R: a, b, c ; \rho ; \eta)= & \int_{1}^{\infty} d u_{1} \ldots \int_{1}^{u_{n-k-1}} d u_{n-k} \int_{0}^{\eta} d u_{n-k+1} \ldots \int_{0}^{u_{n-2}} d u_{n-1} R^{(n)}\left(u_{i}\right) \prod_{i<j}^{n-1}\left(u_{i}-u_{j}\right)^{2 \rho} \\
& \times \prod_{i=1}^{n-k} u_{i}^{a \rho}\left(u_{i}-\eta\right)^{b \rho}\left(u_{i}-1\right)^{c \rho} \prod_{i=n-k+1}^{n-1} u_{i}^{a \rho}\left(\eta-u_{i}\right)^{b \rho}\left(1-u_{i}\right)^{c \rho}
\end{aligned}
$$


and the normalization constants [16]

$$
\begin{aligned}
N_{k}^{(n)}(R: a, b, c ; \rho)= & (-1)^{n-k} \frac{(2 j) !(n-k) !}{(2 j-k+1) !} \prod_{i=0}^{n-k-1} \frac{\Gamma(-\rho(a-2-i)) \Gamma(1+\rho(c+i))}{\Gamma(1+\rho(-a+c+n-k+1+i))} \\
& \times \prod_{i=0}^{k-2} \frac{\Gamma(\rho(a+i)) \Gamma(1+\rho(b+i))}{\Gamma(1+\rho(a+b+k-2+i))} \prod_{i=1}^{n-k} \frac{\Gamma(i \rho)}{\Gamma(\rho)} \prod_{i=1}^{k-1} \frac{\Gamma(i \rho)}{\Gamma(\rho)}
\end{aligned}
$$

We use the notations, $z_{i}-z_{j}=z_{i j}, \eta=\left(z_{12} z_{34}\right) /\left(z_{13} z_{24}\right)$, and $\eta$ is taken to lie on the real axis between 0 and 1 . Other values of $\eta$ can be reached by analytic continuation. In (C.4) $R^{(n)}\left(u_{i}\right)$ is a rational function of the $n-1$ variables $u_{i}$ given by

$$
R^{(n)}\left(u_{i}\right)=(n-1) ! \sum_{p=0}^{n-1} \sum_{\left\{\sigma_{i}\right\}^{p}}\left(\begin{array}{c}
2 j \\
p
\end{array}\right)\left\{\left(u_{1}-\sigma_{1}\right) \ldots\left(u_{n-1}-\sigma_{n-1}\right)\right\}^{-1}
$$

where the second sum runs over all sets of parameters $\sigma_{i}$ such that $p$ of them have the value 0 and $n-1-p$ of them have the value 1 . Note that when $R=1$, the above formulas for the integrals and their normalizations reduce to the expressions (A4) and (A5) derived for the minimal model in Appendix A.

These formulas describe conformal blocks which are correctly normalized for the field at $z_{1}$ fusing with the field at $z_{2}$. This follows from the fact that in the $z_{1} \rightarrow z_{2}(\eta \rightarrow 0)$ limit

$$
I_{k}^{(n)}(R: a, b, c ; \rho ; \eta) \rightarrow N_{k}^{(n)}(R: a, b, c ; \rho) \eta^{\Delta_{j+l-k+1}-\Delta_{j}-\Delta_{l}} f(\eta)
$$

where $f$ is some analytic function of $\eta$ which satisfies $f(0)=1$. The fusion matrix $\alpha$ relates the above conformal blocks to those which are appropriately normalized as $z_{2} \rightarrow z_{3}(\eta \rightarrow 1)$. We calculate the relation between these two sets of blocks precisely as in the minimal model case, explained in Appendix A, by changing variables in $I_{k}^{(n)}$ to $\widetilde{\eta}=1-\eta$ and $\widetilde{u}_{i}=1-u_{i}$. Since $R^{(n)}$ is invariant under these 
changes of variables, we can write

$$
I_{k}^{(n)}(R: a, b, c ; \rho ; \eta)=\widetilde{\alpha}_{k j}^{(n)}(a, b, c ; \rho) I_{j}^{(n)}(R: c, b, a ; \rho ; 1-\eta),
$$

where $\widetilde{\alpha}_{k j}^{(n)}$ has the same form as found in Appendix A (A11) for the minimal series, but with

$$
x=\mathrm{e}^{\mathrm{i} \pi \rho}=-\widetilde{x} .
$$

in place of $\widetilde{x}$.

From the complete expression (C.3) for the conformal blocks and the normalization of the $I_{k}^{(n)}$ 's (C.7), we see that the fusion matrix $\alpha$ is given by

$$
\alpha_{k j}^{(n)}(R: a, b, c ; \rho)=\left\{N_{k}^{(n)}(R: a, b, c ; \rho)\right\}^{-1} \widetilde{\alpha}_{k j}^{(n)}(a, b, c ; \rho) N_{j}^{(n)}(R: c, b, a ; \rho) .
$$

Note that if $a=c, \alpha$ differs from $\widetilde{\alpha}$ by a similarity transformation and so has the same eigenvalues. When $a=c$, by (A11) $\widetilde{\alpha}$ is purely even or odd in $x$. Thus the difference (C.9) between the $S U(2)_{K}$ and minimal model cases is at most an overall sign. Recalling the definitions of the parameters $a, b, c$, and $n$ for the minimal model given in Appendix A, we see that the fusion matrix of the four-point function of spin- $j$ WZW primaries differs from that of the $\phi_{2 j+1,1}$ minimal model primary field by a similarity transformation involving the normalization constants. 


\section{REFERENCES}

1. A.B. Zamolodchikov, Theor. Math. Phys. 65 (1985), 1205.

2. V.A. Fateev and S.L. Lykyanov, Int. J. Mod. Phys. A3 (1988), 507; A. Bilal and J.-L. Gervais, Phys. Lett. 206B (1988), 412, Nucl. Phys. B318 (1989), 579 .

3. A.B. Zamolodchikov and V.A. Fateev, Sov. Phys. J.E.T.P. 62 (1985), 215.

4. V.A. Fateev and A.B. Zamolodchikov, Theor. Math. Phys. 71 (1988), 451.

5. D. Kastor, E. Martinec and Z. Qiu, Phys. Lett. 200B (1988), 434.

6. J. Bagger, D. Nemeschansky and S. Yankielowicz, Phys. Rev. Lett. 60 (1988), 389.

7. F. Ravanini, Mod. Phys. Lett. A3 (1988), 397.

8. G. Felder, Nucl. Phys. B317 (1989), 215.

9. S. Chung, E. Lyman and S.-H. H. Tye, "Fractional Supersymmetry and Minimal Coset Models in Conformal Field Theory," Cornell preprint CLNS $91 / 1057$.

10. C. Ahn, S. Chung and S.-H. H. Tye, "New Parafermion, SU(2) Coset, and N=2 Superconformal Field Theories," Cornell preprint CLNS 91/1053.

11. A.B. Zamolodchikov, LOMI preprint, Lectures in Beijing Summer School, 1989.

12. C. Ahn, D. Bernard and A. LeClair, Nucl. Phys. B346 (1990), 409, and references therein.

13. P. Argyres, A. LeClair and S.-H. H. Tye, Phys. Lett. 253B (1991), 306.

14. P. Argyres and S.-H.H. Tye, Cornell preprint CLNS 91/1068, "Fractional Superstrings with Space-Time Critical Dimensions Four and Six," July 1991.

15. A.B. Zamolodchikov and V.A. Fateev, Sov. J. Nucl. Phys. 43 (1986), 657. 
16. Vl.S. Dotsenko, Nucl. Phys. B338 (1990), 747; Landau Inst. preprint, October, 1990.

17. A.B. Zamolodchikov and R. Poghossian, Sov. J. Nucl. Phys. 47 (1988), 1461.

18. R. Poghossian, Int. J. Mod. Phys. 6A (1991), 2005.

19. G. Moore and N. Seiberg, Phys. Lett. 212B (1988), 451, Nucl. Phys. B313 (1989), 16, Commun. Math. Phys. 123 (1989), 177.

20. G. Felder, J. Fröhlich and G. Keller, Commun. Math. Phys. 124 (1989), 417, Commun. Math. Phys. 130 (1990), 1.

21. A.A. Belavin, A.M. Polyakov and A.B. Zamolodchikov, Nucl. Phys. B241 (1984), 333 .

22. M. Wakimoto, Commun. Math. Phys. 104 (1986), 604.

23. Vl.S. Dotsenko and V.A. Fateev, Nucl. Phys. B240 (1984), 312, Nucl.Phys. B251 (1985), 691.

24. G. Felder, J. Fröhlich and G. Keller, Commun. Math. Phys. 124 (1989), 647.

25. B.L. Feigin and D.B. Fuchs, Funct. Anal. Appl. 16 (1982), 114; G. Felder, Nucl. Phys. B317 (1989), 215.

26. D. Bernard and A. LeClair, Phys. Lett. 247B (1990), 309.

27. D. Friedan, Z. Qiu and S. Shenker, Phys. Rev. Lett. 52 (1984), 1575.

28. A. Cappelli, C. Itzykson and J.-B. Zuber, Nucl. Phys. B280 (1987), 445.

29. F. Ravanini, Mod. Phys. Lett. A3 (1988), 271.

30. See e.g. K. Gottfried, Quantum Mechanics Volume I: Fundamentals, BenjaminCummings, Reading, Massachusetts (1966). 


\section{TABLE CAPTIONS}

1: Realizations of known representations of the $\mathcal{A}^{(K)}$ fractional supersymmetry chiral algebra in terms of "free" fields. $\left(\varphi, \alpha_{0}\right)$ stands for a boson with background charge, and $\left(\omega, \omega^{+}\right)$the $c=2$ first order bosonic free field system.

\section{FIGURE CAPTIONS}

1) The action of fusion transformations $\alpha$ on conformal blocks.

2) The action of braiding transformations $\beta$ on conformal blocks.

3) Associativity constraint for the $\phi_{3,1}$ four-point correlation function in the $K=4$ minimal model.

4) The action of twisting transformations $\gamma$ on conformal blocks.

5) Illustration of the relation $\beta=\alpha \gamma \alpha$ (5.2) relating the braiding transformation to two fusions and a twist.

6) Illustration of the relation $\beta=\gamma \alpha \gamma$ (A12) relating the braiding transformation to two twists and a fusion.

\begin{tabular}{|c|c|c|c|}
\hline Representation of $\mathcal{A}^{(K)}$ & Central charge & 'Free' field realization & $J^{(K)}$ current \\
\hline$K$ th minimal model & $c_{\min }=\frac{K(K+5)}{(K+2)(K+3)}$ & $\left(\varphi, \alpha_{0}\right)$ & $\phi_{3,1}$ \\
$\frac{S U(2)_{K} \otimes S U(2)_{L}}{S U(2)_{K+L}}$ cosets & $c_{\min } \leq c \leq c_{S U(2)}$ & $\left(\varphi, \alpha_{0}\right)+Z_{K}$-parafermion & $\epsilon_{1} \partial \varphi+\ldots(3.7)$ \\
$S U(2)_{K}$ WZW model & $c_{S U(2)}=\frac{3 K}{K+2}$ & $\left(\varphi, \alpha_{0}\right)+\left(\omega, \omega^{+}\right)$ & $q_{a b} J_{-1}^{a} \Phi_{(1)}^{b}$ \\
\hline
\end{tabular}

Table 1 . 